\title{
$U$ (1) extension of the standard model from short-distance structure of spacetime
}

\author{
Cao H. Nam ${ }^{1,2, a}$ \\ ${ }^{1}$ Institute of Research and Development, Duy Tan University, Da Nang 550000, Vietnam \\ 2 Department of Physics, College of Science, Yonsei University, Seoul 120-749, Korea
}

Received: 26 December 2018 / Accepted: 20 April 2019 / Published online: 3 May 2019

(C) The Author(s) 2019

\begin{abstract}
In this paper, we presented a U(1) extension of the SM and the corresponding consequences, based on a more fundamental structure of the spacetime. We started fundamentally from a generally covariant theory which includes a set of the fields propagating dynamically in the fundamental spacetime and respecting for the SM gauge group. We then derived, in the effective four-dimensional spacetime, an extension of the SM with the gauge group $\mathrm{SU}(3)_{C} \otimes$ $\mathrm{SU}(2)_{L} \otimes \mathrm{U}(1)_{Y} \otimes \mathrm{U}(1)_{X}$. Due to the structure of the spacetime, the tiny observed neutrino masses are an unavoidable consequence in this scenario. Also, the phenomenology of the new neutral gauge boson is discussed in detail.
\end{abstract}

\section{Introduction}

The present experimental observations indicate that the spacetime is a four-dimensional manifold. However, it has been believed that the presently observed spacetime is actually not fundamental because of some reasons. For example, the gravitational interaction results in the unphysical black hole and big bang singularities at which the curvature scalars of the spacetime, densities become infinite [1]. In other words, the spacetime itself may possess more complex and deeper structures at high energy regions or short distances, at which new dynamical degrees of freedom of the spacetime should be exhibited significantly. The extension of the spacetime was first proposed by Kaluza and Klein in attempting to show that a Abelian gauge field can emerge in the four-dimension spacetime from the metric of the fivedimensional spacetime $[2,3]$. It was generalized to greater dimensionality to obtain the non-Abelian gauge fields [49] (also see Refs. [10,11] for reviews). In recent years, the extension of the spacetime has attracted enormous attention because of its phenomenology aspect [12-19].

\footnotetext{
a e-mail: hncao@yonsei.ac.kr
}

Our present understanding of the elementary particles and their interactions is based on the standard model (SM) whose particle content has been completely confirmed by the LHC collaborations ATLAS and CMS [20,21]. Its gauge symmetry group is $\mathrm{SU}(3)_{C} \otimes \mathrm{SU}(2)_{L} \otimes \mathrm{U}(1)_{Y}$ corresponding to the spin-1 particles: the gluons, W/Z bosons, and photon which are responsible for mediating the strong, weak, and electromagnetism forces, respectively. An important question is whether there are additional short-range gauge forces. In particular, new short-range Abelian gauge forces are the simplest extensions of the SM. They appear in grand unified theories (GUTs) [22-28], in string theoretical models [29-32], in little Higgs models [33-35], in theories with extra dimensions [17,36-40], and in various extensions [41-66]. (Also, the readers can see Refs. [67-69] for reviews.) The search for such gauge forces is an active area at the LHC [70-76] and future colliders such as ILC. Usually, introducing an additional Abelian gauge force is through the extension of the SM gauge symmetry group by adding an additional, fundamental U(1) symmetry to the theory However, there has still the possibility that the new Abelian gauge force comes from a more complex and deeper structure of the spacetime. This is motivated by the fact that one of presently well-known four fundamental forces, the gravitational interaction, arises as a result of the geometric structure of the spacetime.

In this paper, we would like to show that an additional $\mathrm{U}(1)$ symmetry can be emerged as a result of short-distance structure of the spacetime. The U(1) extension of the SM in this paper is distinguishable from the usual U(1) extensions of the SM, such as the $\mathrm{U}(1)_{B-L}$ extension. First, the additional $U(1)$ charges of the SM fermions in this paper are different to those of the SM fermions in the usual U(1) extensions of the SM, because here the right-handed neutrinos do not carry the charges under this Abelian symmetry. [Whereas, in the usual U(1) extensions of the SM, the righthanded neutrinos need to carry the additional $\mathrm{U}(1)$ charges. This is necessary to generate the heavy Majorana masses 
for the right-handed neutrinos after the spontaneous breaking.] Second, the gauge boson associated with the additional $\mathrm{U}(1)$ has both usual and unusual couplings. With respect to the unusual coupling, more specifically, this gauge boson can couple to a particle and an anti-particle of another field. Also, it is interesting that the small masses of the neutrinos are an unavoidable consequence of the short-distance structure of the spacetime, without imposing the Yukawa couplings between the right-handed neutrinos and exotic Higgs to get heavy Majorana masses like the usual U(1) extensions. In addition, the atomic parity violation in Cesium $\left({ }_{55}^{133} \mathrm{Cs}\right)$ constrains very strongly on the spontaneous symmetry breaking scale of the additional U(1), with a lower bound to be about $7 \mathrm{TeV}$. Thus, it is testable at current and future colliders.

This paper is organized as follows. In Sect. 2, we propose a more fundamental structure for the spacetime and discuss relevant important properties. In Sect. 3, we build a realistic model at which we present the particle content, the spontaneous symmetry breaking, the mass spectrum, and phenomenology of the new gauge boson. Finally, we devote to conclusion in the last section. In this work, we use units in $\hbar=c=1$ and the signature of the metric $(+,-,-,-, \ldots)$.

\section{Setups}

In this section, we would like to propose a more fundamental structure for the spacetime, which is expected to exhibit at (very) short distances or high energies. Then, we discuss relevant important properties, based on which in next section we will build a realistic model with the gauge symmetry group $\mathrm{SU}(3)_{C} \otimes \mathrm{SU}(2)_{L} \otimes \mathrm{U}(1)_{Y}$.

\subsection{Fundamental spacetime}

We assume that, at more fundamental level, the spacetime is a five-dimensional fiber bundle $M_{5}$, shown illustratively in Fig. 1 , whose base manifold and fiber are the four-dimensional Minkowski-flat manifold $M_{4}$ and the Lie group manifold $\mathrm{U}(1)$, respectively. ${ }^{1}$ By definition, the spacetime $M_{5}$ consists of the following elements [78]:

1. A surjective projection $\pi: M_{5} \longrightarrow M_{4}$. Each inverse image $\pi^{-1}(x) \cong \mathrm{U}(1)$ is a submanifold of $M_{5}$ and called a fiber.

\footnotetext{
${ }^{1}$ Because the fact that the usual $4 D$ gravitation taken into account does not affect the aim of the present work, for simplicity we consider the extension of the $4 D$ Minkowski-flat spacetime. In the case of that the usual $4 D$ gravitation is included, the base manifold should be no longer flat but curved.
}

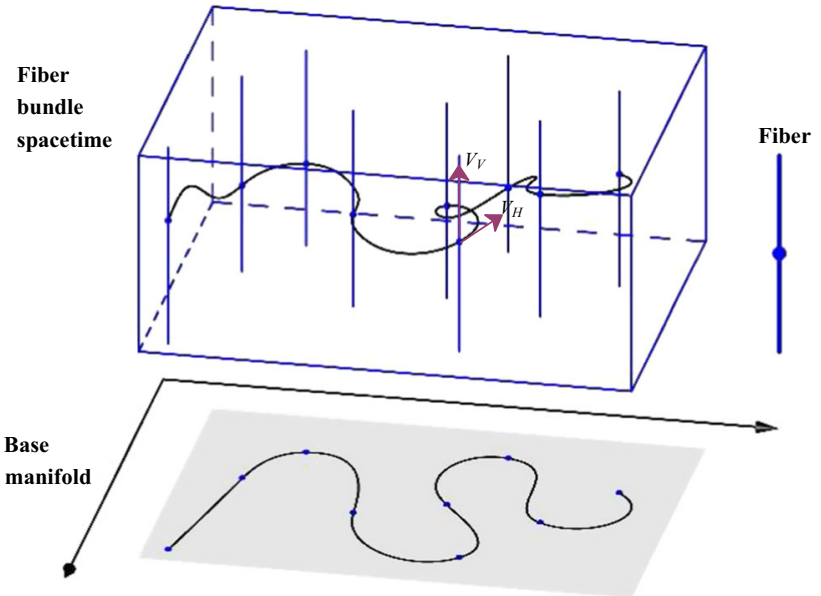

Fig. 1 An illustrative figure for the fiber bundle spacetime. There has a surjective projection $\pi$ from the fiber bundle spacetime to the base manifold, and each inverse image $\pi^{-1}(x) \cong \mathrm{U}(1)$ is a submanifold of the spacetime and called a fiber. Any tangent vector at a point in the fiber bundle spacetime is always decomposed into a sum, $V_{V}+V_{H}$, where $V_{V}$ is tangent to the fiber and $V_{H}$ is to point from one fiber to another. Note that, the original figure is given in Ref. [77]

2. In general, the spacetime $M_{5}$ is covered by a set of charts $\left\{\left(V_{i} \times \mathrm{U}(1), \phi_{i}\right)\right\}$, where $V_{i}$ is a local region (open subset) of $M_{4}$ and $\phi_{i}$ is a diffeomorphism map as $\phi_{i}: V_{i} \times$ $\mathrm{U}(1) \longrightarrow \pi^{-1}\left(V_{i}\right)$ [where $\pi^{-1}\left(V_{i}\right)$ is of course a local region of the spacetime $M_{5}$ ].

In this sense, one local region of the spacetime $M_{5}$ looks like a product $V_{i} \times \mathrm{U}(1)$. Since the chart $\left(V_{i} \times \mathrm{U}(1), \phi_{i}\right)$ enables to give the local coordinates for a point in the spacetime $M_{5}$ as, $\left(x^{\mu}, e^{i \theta}\right)$, where $\left\{x^{\mu}\right\} \in V_{i} \subset M_{4}$ and $e^{i \theta} \in \mathrm{U}(1)$ with $\theta$ to be dimensionless real parameter. If any two charts $\left(V_{i} \times \mathrm{U}(1), \phi_{i}\right)$ and $\left(V_{j} \times \mathrm{U}(1), \phi_{j}\right)$ overlap together, we have

$p=\phi_{i}\left(x, e^{i \theta}\right)=\phi_{j}\left(x, e^{i \theta^{\prime}}\right)$

where $p$ refers to a point in the spacetime $M_{5}$. This leads to the following general coordinate transformation

$x^{\mu} \longrightarrow x^{\prime \mu}=x^{\mu}$,

$e^{i \theta} \longrightarrow e^{i \theta^{\prime}}=h(x) e^{i \theta}$, or $\theta \longrightarrow \theta^{\prime}=\theta+\alpha(x)$,

where $h(x)=\phi_{j, x}^{-1} \circ \phi_{i, x}$ and $\alpha(x)=-i \ln [h(x)]$. [Note that, there has no $x$-coordinate change because the base manifold of the spacetime $M_{5}$ is flat.] The theory is covariant with respect to this general coordinate transformation.

Of important properties of the spacetime $M_{5}$ is that there exists naturally the active action of the Lie group $\mathrm{U}(1)$ on it (which turns a point of $M_{5}$ into another point of $M_{5}$, not to change the local coordinates of the same point), defined as [78] 


$$
\begin{aligned}
& R_{a}: M_{5} \longrightarrow M_{5}, \\
& \phi_{i}\left(x, e^{i \theta}\right)=p \longmapsto p a=\phi_{i}\left(x, e^{i \theta}\right) a=\phi_{i}\left(x, e^{i \theta} a\right),
\end{aligned}
$$

where $a=e^{i \beta}$ is any element of the Lie group $\mathrm{U}(1)$. Indeed, the definition of the active action (3) is independent on the choice of the chart or local coordinates, since

$p a=\phi_{j}\left(x, e^{i \theta^{\prime}} a\right)=\phi_{j}\left(x, h(x) e^{i \theta}\right) a=\phi_{i}\left(x, e^{i \theta} a\right)$,

where it should be noted $h(x)=\phi_{j, x}^{-1} \circ \phi_{i, x}$. Thus, the active action of the Lie group $\mathrm{U}(1)$ on the spacetime $M_{5}$ appears in a natural way. Interestingly, for $\Phi\left(x, e^{i \theta}\right)$ to be a field on the spacetime $M_{5}$, it can define the active action of the Lie group $\mathrm{U}(1)$ on the field $\Phi$, which is induced by the active action of the Lie group U(1) on the spacetime $M_{5}$. More specifically, this action is defined as

$$
\begin{aligned}
R_{a}: M_{5} & \longrightarrow M_{5}, \\
\phi_{i}\left(x, e^{i \theta}\right)=p & \longmapsto p a=\phi_{i}\left(x, e^{i \theta} a\right), \\
T_{a}:\left.\operatorname{Vspace}(\Phi)\right|_{p} & \left.\longrightarrow \operatorname{Vspace}(\Phi)\right|_{p a}, \\
\left.\Phi\right|_{\left(x, e^{i \theta}\right)} & \left.\longmapsto \Phi\right|_{\left(x, e^{i \theta}\right)} T[a],
\end{aligned}
$$

where $\left.\operatorname{Vspace}(\Phi)\right|_{p}$ and $\left.\operatorname{Vspace}(\Phi)\right|_{p a}$ are denoted to the value spaces of the field $\Phi$ at the points $p$ and $p a$, respectively, $\left.\Phi\right|_{\left(x, e^{i \theta}\right)}$ refers to the value of the field $\Phi$ at the point $p$ of the local coordinates $\left(x, e^{i \theta}\right)$, and $T[a]$ is a representation of the element $a=e^{i \beta}$, which is of course dependent on the field $\Phi$, given as

$T\left[e^{i \beta}\right]=e^{i X_{\Phi} \beta}$,

with $X_{\Phi}$ to be a quantum number characterizing the active action of the Lie group $\mathrm{U}(1)$ on the field $\Phi$. Note that, $\left.\operatorname{Vspace}(\Phi)\right|_{p}$ and Vspace $\left.(\Phi)\right|_{p a}$ should not be confused with the function space of the field $\Phi$ on the spacetime $M_{5}$. In general, $\left.\Phi\right|_{\left(x, e^{i \theta}\right)} T[a]$ should not match the value of the field $\Phi$ at the point $p a$ of the local coordinates $\left(x, e^{i \theta} a\right)$, meaning that

$\left.\Phi\right|_{\left(x, e^{i \theta} a\right)} \neq\left.\Phi\right|_{\left(x, e^{i \theta}\right)} T[a]$.

On the contrary, if $\left.\Phi\right|_{\left(x, e^{i \theta}\right)} T[a]$ matches the value of the field $\Phi$ at the point $p a$, we have

$\left.\Phi\right|_{\left(x, e^{i \theta} a\right)}=\left.\Phi\right|_{\left(x, e^{i \theta}\right)} T[a]$.

If the identity (8) holds for arbitrary $\left(x, e^{i \theta}\right)$ and $a$, then the field $\Phi$ is called to be invariant under the active action of the Lie group U(1). It is clearly that (6) and (8) [holding for arbitrary $\left(x, e^{i \theta}\right)$ and $a$ ] imply a specific function form for the field $\Phi$ which is invariant under the active action of the Lie group $\mathrm{U}(1)$, as
$\Phi\left(x, e^{i \theta}\right)=\phi(x) e^{i X_{\Phi} \theta}$

where $\phi(x)$ describes the $x$-dependence of $\Phi$. In this way, with respect to a field which is invariant under the active action of the Lie group $U(1)$, it itself has a specific $\theta$ dependence. Under the general coordinate transformation (2), we have $\Phi^{\prime}\left(x^{\prime}, e^{i \theta^{\prime}}\right)=\Phi\left(x, e^{i \theta}\right)$, thus the $x$-dependence of $\Phi$ must transform as

$\phi(x) \longrightarrow \phi^{\prime}(x)=e^{-i X_{\Phi} \alpha(x)} \phi(x)$,

which is nothing but the usual local gauge transformation.

\subsection{Gauge field from the structure of spacetime $M_{5}$}

In this subsection, we show that the structure of the spacetime $M_{5}$ leads naturally to the existence of a definite field which transforms under the general coordinate transformation (2) in the rule of the usual gauge transformation. Then, we determine how the fundamental quantities of the spacetime $M_{5}$ are expressed in terms of this field. Finally, we obtain the kinetic term of this field from the action for the spacetime $M_{5}$ or the Einstein-Hilbert (EH) action.

In order to do these, we begin with an intrinsic property of the spacetime $M_{5}$. Because of the fiber bundle structure, any tangent vector $V$ of the spacetime $M_{5}$ is always decomposed into two orthogonal components (as shown in Fig. 1), independently on the choice of the local coordinate system, as

$V=V_{H}+V_{V}$

Here, $V_{V}$ is tangent to the fiber and so-called the vertical tangent vector. And, $V_{H}$ is the complement of the vertical tangent vector $V_{V}$, which points from one fiber to another and so-called the horizontal tangent vector. In this way, the tangent space $T_{p} M_{5}$ at any point $p$ of the spacetime $M_{5}$ is always decomposed into a direct sum of two subspaces as

$T_{p} M_{5}=H_{p} M_{5} \oplus V_{p} M_{5}$,

where $H_{p} M_{5}$ and $V_{p} M_{5}$ are (four-dimensional) horizontal and (one-dimensional) vertical tangent subspaces which define the horizontal and vertical directions at the point $p$ of the spacetime $M_{5}$, respectively. It can easily see that the vertical tangent subspace is spanned by $\frac{\partial}{\partial \theta} \equiv \partial_{\theta}$. This is because under the general coordinate transformation (2) $\partial_{\theta}$ transforms in a covariant way as

$\frac{\partial}{\partial \theta} \longrightarrow \frac{\partial}{\partial \theta^{\prime}}=\frac{\partial x^{\mu}}{\partial \theta^{\prime}} \frac{\partial}{\partial x^{\mu}}+\frac{\partial \theta}{\partial \theta^{\prime}} \frac{\partial}{\partial \theta}=\frac{\partial \theta}{\partial \theta^{\prime}} \frac{\partial}{\partial \theta}$.

Whereas, the horizontal tangent subspace is not spanned by $\left\{\frac{\partial}{\partial x^{\mu}} \equiv \partial_{\mu}\right\}$, because the change of $\left\{\partial_{\mu}\right\}$ under the general coordinate transformation (2) as 


$$
\begin{aligned}
\frac{\partial}{\partial x^{\mu}} \longrightarrow \frac{\partial}{\partial x^{\prime \mu}} & =\frac{\partial x^{\nu}}{\partial x^{\prime \mu}} \frac{\partial}{\partial x^{\nu}}+\frac{\partial \theta}{\partial x^{\prime \mu}} \frac{\partial}{\partial \theta} \\
& =\frac{\partial x^{\nu}}{\partial x^{\prime \mu}}\left(\frac{\partial}{\partial x^{\nu}}-\partial_{\nu} \alpha(x) \frac{\partial}{\partial \theta}\right),
\end{aligned}
$$

is not covariant due to an extra term relevant to $\partial_{\theta}$ which does not belong to $\left\{\partial_{\mu}\right\}$. Clearly, for a covariant basis of the horizontal tangent subspace, it requires a definite field whose change under the general coordinate transformation (2) compensates the change of $\partial_{\mu}$. In appendix, we show that a covariant basis of the horizontal tangent subspace is defined as

$$
\left\{\frac{\partial}{\partial x^{\mu}}-g_{X} X_{\mu} \frac{\partial}{\partial \theta} \equiv \hat{\partial}_{\mu}\right\},
$$

where the field $X_{\mu}$ with the dimension $\left[X_{\mu}\right]=1$ transforms under the general coordinate transformation (2) in the following rule

$X_{\mu} \longrightarrow X_{\mu}^{\prime}=X_{\mu}-\frac{1}{g_{X}} \partial_{\mu} \alpha(x)$,

and $g_{X}$ is a dimensionless constant which plays the role of the coupling constant characterizing the strength of the interaction mediated by $X_{\mu}$. Indeed, $\left\{\hat{\partial}_{\mu}\right\}$ is a covariant basis of the horizontal tangent subspace, because under the general coordinate transformation $\hat{\partial}_{\mu}$ transforms as follows

$\hat{\partial}_{\mu} \longrightarrow \hat{\partial}_{\mu}^{\prime}=\frac{\partial x^{\nu}}{\partial x^{\prime \mu}} \hat{\partial}_{\nu}$

The commutator of two basic vectors of the horizontal tangent subspace defines the field strength of the gauge field $X_{\mu}$ as

$\left[\hat{\partial}_{\mu}, \hat{\partial}_{\nu}\right]=-g_{X} X_{\mu \nu} \partial_{\theta}$,

where $X_{\mu \nu}=\partial_{\mu} X_{v}-\partial_{v} X_{\mu}$.

In summary, the tangent space of the spacetime $M_{5}$ is spanned by $\left\{\left(\hat{\partial}_{\mu}, \partial_{\theta}\right)\right\} \equiv\left\{\partial_{M}\right\}$. The commutator of any two frame fields is

$\left[\partial_{M}, \partial_{N}\right]=C_{M N}^{P} \partial_{P}$,

where $C_{M N}^{P}$ are the non-holonomic functions. The explicit expressions of $C_{M N}^{P}$ are determined as follows

$$
\begin{aligned}
& C_{\mu \nu}^{\lambda}=0, \quad C_{\mu \nu}^{\theta}=-g_{X} X_{\mu \nu}, \quad C_{\mu \theta}^{\nu}=-C_{\theta \mu}^{\nu}=0, \\
& C_{\mu \theta}^{\theta}=-C_{\theta \mu}^{\theta}=0, \quad C_{\theta \theta}^{\theta}=0 .
\end{aligned}
$$

A dual vector space to $T_{p} M_{5}$ is called the cotangent space, denoted by $T_{p}^{*} M_{5}$, which is always decomposed into a direct sum of two subspaces as

$$
T_{p}^{*} M_{5}=V_{p}^{*} M_{5} \oplus H_{p}^{*} M_{5},
$$

where $H_{p}^{*} M_{5}$ and $V_{p}^{*} M_{5}$ are horizontal and vertical cotangent subspaces, which are dual to the horizontal tangent subspace $H_{p} M_{5}$ and the vertical one $V_{p} M_{5}$, respectively. The covariant bases of the dual subspaces $H_{p}^{*} M_{5}$ and $V_{p}^{*} M_{5}$ are $\left\{d x^{\mu}\right\}$ and $\left\{d \theta+g_{X} X_{\mu} d x^{\mu}\right\}$, respectively. Indeed, we can easily show that they transform under the general coordinate transformation (2) as

$$
\begin{aligned}
d x^{\mu} & \longrightarrow d x^{\prime \mu}=\frac{\partial x^{\prime \mu}}{\partial x^{\nu}} d x^{\nu}, \\
d \theta+g_{X} X_{\mu} d x^{\mu} & \longrightarrow d \theta^{\prime}+g_{X} X_{\mu}^{\prime} d x^{\prime \mu} \\
& =\frac{\partial \theta^{\prime}}{\partial \theta}\left(d \theta+g_{X} X_{\mu} d x^{\mu}\right),
\end{aligned}
$$

which are clearly covariant. Also, they are dual bases, since

$$
\begin{aligned}
\left\langle d x^{\mu}, \hat{\partial}_{\nu}\right\rangle & =\frac{\partial x^{\mu}}{\partial x^{\nu}}-g_{X} X_{v} \frac{\partial x^{\mu}}{\partial \theta}=\frac{\partial x^{\mu}}{\partial x^{\nu}}=\delta_{v}^{\mu}, \\
\left\langle d \theta+g_{X} X_{\mu} d x^{\mu}, \partial_{\theta}\right\rangle & =\frac{\partial \theta}{\partial \theta}+g_{X} X_{\mu} \frac{\partial x^{\mu}}{\partial \theta}=\frac{\partial \theta}{\partial \theta}=1 \\
\left\langle d x^{\mu}, \partial_{\theta}\right\rangle & =\frac{\partial x^{\mu}}{\partial \theta}=0 \\
\left\langle d \theta+g_{X} X_{\mu} d x^{\mu}, \hat{\partial}_{\nu}\right\rangle & =\frac{\partial \theta}{\partial x^{\nu}}+g_{X} X_{\mu} \frac{\partial x^{\mu}}{\partial x^{\nu}}-g_{X} X_{v} \frac{\partial \theta}{\partial \theta} \\
-g_{X}^{2} X_{\mu} X_{v} \frac{\partial x^{\mu}}{\partial \theta} & =0
\end{aligned}
$$

where the action of any one-form $d f \in T_{p}^{*} M_{5}$ on any vector $V \in T_{p} M_{5}$ is defined by

$\langle d f, V\rangle \equiv V[f]=V^{\mu} \frac{\partial f}{\partial x^{\mu}}+V^{\theta} \frac{\partial f}{\partial \theta}$.

In order to introduce the action for the spacetime $M_{5}$ or the $\mathrm{EH}$ action, first let us define a metric or an invariant distance element $G$ between nearby points on the spacetime $M_{5}$. For $X$ and $Y$ to be any two tangent vectors of the spacetime $M_{5}$, their inner product is defined by the metric $G$ as

$G\left(V_{1}, V_{2}\right)=G_{H}\left(V_{1 H}, V_{2 H}\right)+G_{V}\left(V_{1 V}, V_{2 V}\right)$,

where $V_{1 H}\left(V_{2 H}\right)$ and $V_{1 V}\left(V_{2 V}\right)$ are the horizon and vertical components of the vector $V_{1}\left(V_{2}\right)$, respectively. Of course, the inner product between any horizontal tangent vector and vertical one is zero because they belong to two orthogonal subspaces. In this sense, the metric $G$ on the spacetime $M_{5}$ is specified by the pair $\left(G_{H}, G_{V}\right)$. Here, $G_{H}$ is the horizontal metric which defines the inner product between any vectors belonging the horizontal tangent subspace, and $G_{V}$ is the vertical metric which defines the inner product between any vectors belonging the vertical tangent subspace. On the other hand, $G_{H}$ defines the invariant distance element between nearby points along the horizontal directions, whereas $G_{V}$ defines the invariant distance element between nearby points along the vertical directions. The horizontal metric $G_{H}$ is a tensor field whose value at any point $p \in M_{5}$ belongs the 
space $H_{p}^{*} M_{5} \otimes H_{p}^{*} M_{5}$, which is naturally defined by

$G_{H}=\pi^{*}\left(\eta_{\mu \nu} d x^{\mu} d x^{\nu}\right)=\eta_{\mu \nu} d x^{\mu} d x^{\nu}$,

where $\pi^{*}$ is the pullback map which is naturally induced by the projection operator $\pi$. Note that, the term $\eta_{\mu \nu} d x^{\mu} d x^{\nu}$ in the sign (...) is the metric on the manifold $M_{4}$ which is base manifold of the spacetime $M_{5}$. Whereas, the vertical metric $G_{V}$ is a tensor field whose value at any point $p \in M_{5}$ belongs the space $V_{p}^{*} M_{5} \otimes V_{p}^{*} M_{5}$. Thus, $G_{V}$ is in general defined by

$G_{V}=-T^{2}\left(x, e^{i \theta}\right) \frac{\left(d \theta+g_{X} X_{\mu} d x^{\mu}\right)^{2}}{\Lambda^{2}}$,

where $T\left(x, e^{i \theta}\right)$ is the field determining the geometric size of the fiber and $\Lambda$ is a new constant of the energy dimension which is much higher than present energy scale. Note that, $\left(d \theta+g_{X} X_{\mu} d x^{\mu}\right) / \Lambda$ is the infinitesimal length on the fiber. In this paper, we consider theory at the vacuum $\left\langle T\left(x, e^{i \theta}\right)\right\rangle=$ $T_{0}$. Since, the geometric size of the fibers is determined by the radius $R=T_{0} / \Lambda$.

In the basic $\left\{\left(\hat{\partial}_{\mu}, \partial_{\theta}\right)\right\}\left(\equiv\left\{\partial_{M}\right\}\right)$, the coefficients of the Christoffel connection and the Riemann curvature tensor are defined as

$$
\begin{aligned}
\Gamma_{M N}^{P}= & \frac{G^{P Q}}{2}\left(\partial_{M} G_{N Q}+\partial_{N} G_{M Q}-\partial_{Q} G_{M N}\right) \\
& +\frac{G^{P Q}}{2}\left(C_{Q M}^{O} G_{O N}+C_{Q N}^{O} G_{O M}\right)+\frac{C_{M N}^{P}}{2} \\
\mathcal{R}_{M P N}^{O}= & \partial_{N}\left[\Gamma_{P M}^{O}\right]-\partial_{P}\left[\Gamma_{N M}^{O}\right]+\Gamma_{P M}^{Q} \Gamma_{N Q}^{O} \\
& -\Gamma_{N M}^{Q} \Gamma_{P Q}^{O}+C_{P N}^{Q} \Gamma_{Q M}^{O},
\end{aligned}
$$

where

$G_{M N}=\operatorname{diag}\left(\eta_{\mu \nu},-R^{2}\right), \quad G^{M N}=\operatorname{diag}\left(\eta^{\mu \nu},-1 / R^{2}\right)$.

With the results derived above, the scalar curvature $\mathcal{R}$ of the spacetime $M_{5}$ reads

$$
\begin{aligned}
\mathcal{R} & =G^{M N} \mathcal{R}_{M P N}^{P}=\eta^{\mu \nu} \mathcal{R}_{\mu M \nu}^{M}-\frac{1}{R^{2}} \mathcal{R}_{\theta M \theta}^{M}, \\
& =-\frac{g_{X}^{2} R^{2}}{4} X_{\mu \nu} X^{\mu \nu} .
\end{aligned}
$$

Then, we can find the action for the spacetime $M_{5}$ or the EH action as

$$
\begin{aligned}
S_{E H} & =\frac{M_{*}^{3}}{2} \int d^{4} x d \theta|\operatorname{det} G|^{1 / 2} \mathcal{R}, \\
& =\frac{\left(g_{X} M_{P l} R\right)^{2}}{2} \int d^{4} x\left(-\frac{1}{4} X_{\mu \nu} X^{\mu \nu}\right),
\end{aligned}
$$

where $M_{*}$ is the five-dimensional Planck scale which is related to the four-dimensional one $M_{P l}$ as, $2 \pi R M_{*}^{3}=M_{P l}^{2}$. To get the canonically normalized action for the gauge field
$X_{\mu}$, the coupling constant $g_{X}$ in (31) should be determined as

$g_{X}=\frac{\sqrt{2}}{M_{P l} R}=\frac{\sqrt{2} \Lambda}{T_{0} M_{P l}}$.

This implies that the interacting strength of the gauge field $X_{\mu}$ to other fields should be very small if the inverse bulk radius is much smaller than the Planck scale, $\Lambda / T_{0} \ll M_{P l}$.

\subsection{Fundamental fermions on spacetime $M_{5}$}

We can immediately check that the coordinates $\left(x^{\mu}, e^{i \theta}\right)$ and other $\left(\Lambda^{\mu}{ }_{\nu} x^{\nu}, e^{i \theta}\right)$, with the constant matrix $\Lambda^{\mu}{ }_{\nu}$ to be an element of the Lorentz group $\mathrm{SO}(3,1)$, determine to the same horizontal metric $G_{H}$ and thus leave invariant the spacetime metric. As a result, it is possible to define the usual right-handed and left-handed Weyl spinor fields in the fivedimensional spacetime $M_{5}$. More specifically, a left-handed Weyl spinor field $\Psi_{L}\left(x, e^{i \theta}\right)$ in the five-dimensional spacetime $M_{5}$ is defined as a field that transforms as

$$
\begin{aligned}
\Psi_{L}\left(x^{\mu}, e^{i \theta}\right) & \longrightarrow \Psi_{L}^{\prime}\left(\Lambda_{\nu}^{\mu} x^{\nu}, e^{i \theta}\right) \\
& =\rho_{L}\left(\Lambda^{\mu}{ }_{\nu}\right) \Psi_{L}\left(x^{\mu}, e^{i \theta}\right),
\end{aligned}
$$

where $\rho_{L}\left(\Lambda_{\nu}^{\mu}\right)$ is a matrix corresponding to this representation. Similarly, a right-handed Weyl spinor field $\Psi_{R}\left(x, e^{i \theta}\right)$ transforms with the corresponding matrix $\rho_{R}\left(\Lambda^{\mu}{ }_{\nu}\right)$. Note that, under the general coordinate transformation (2), these spinor fields do not transform or they are scalars, meaning that

$\Psi_{L, R}^{\prime}\left(x^{\prime}, e^{i \theta^{\prime}}\right)=\Psi_{L, R}\left(x, e^{i \theta}\right)$.

For $\Psi\left(x, e^{i \theta}\right)$ to be a right-handed or left-handed Weyl spinor field, let us write invariant action $S[\Psi]$ describing its propagation in the spacetime $M_{5}$. Corresponding to the horizontal derivatives $\hat{\partial}_{\mu}$, invariant term is given by

$S_{H}[\Psi]=\int d x^{4} d \theta \sqrt{|\operatorname{det} G|} \bar{\Psi} i \gamma^{\mu} \hat{\partial}_{\mu} \Psi$,

which governs the dynamics of $\Psi$ along the horizontal directions in the spacetime. Note that, from this first term, one can extract the coupling between $X_{\mu}$ and $\Psi$ as, $-i g_{X} \bar{\Psi} \gamma^{\mu} \partial_{\theta} \Psi X_{\mu}$, where the charge is dynamically generated due to the change of the field $\Psi$ along the vertical direction. Whereas, corresponding to the vertical derivative $\partial_{\theta}$, invariant term is given by 


$$
\begin{aligned}
S_{V}[\Psi]= & \int d x^{4} d \theta \sqrt{|\operatorname{det} G|} \frac{1}{2 \Lambda} \\
& \times\left(\partial^{\theta} \bar{\Psi}^{C} \partial_{\theta} \Psi-M^{2} \bar{\Psi}^{C} \Psi+\text { H.c. }\right), \\
= & \int d x^{4} d \theta \sqrt{|\operatorname{det} G|} \frac{1}{2 \Lambda} \\
& \times\left[\partial^{\theta} \Psi^{T} C \partial_{\theta} \Psi+\partial^{\theta} \bar{\Psi} C \partial_{\theta} \bar{\Psi}^{T}\right. \\
& \left.-M^{2}\left(\Psi^{T} C \Psi+\bar{\Psi} C \bar{\Psi}^{T}\right)\right]
\end{aligned}
$$

that governs the dynamics of $\Psi$ along the vertical direction in the spacetime $M_{5}$. Here, $\partial^{\theta}=G_{V}^{\theta \theta} \partial_{\theta}=-\partial_{\theta} / R^{2}, M$ is the vertical mass parameter which is naturally the order of the scale $\Lambda$, and $\Psi^{C}=C \bar{\Psi}^{T}$ with $T$ denoting the transpose and $C=i \gamma^{2} \gamma^{0}$ to be the charge conjugation operator satisfying the relations $C^{-1}=C^{\dagger}=C^{T}=-C$ and $C^{-1} \gamma^{\mu} C=-\left(\gamma^{\mu}\right)^{T}$. Note that, the vertical mass parameter can be chosen to be real without loss of generality. Then, invariant action $S[\Psi]$ describing the propagation of the field $\Psi$ in the spacetime $M_{5}$ reads

$$
\begin{aligned}
S[\Psi]= & S_{H}[\Psi]+S_{V}[\Psi], \\
= & \int d x^{4} d \theta \sqrt{|\operatorname{det} G|} \\
& {\left[\bar{\Psi} i \gamma^{\mu} \hat{\partial}_{\mu} \Psi\right.} \\
& \left.+\frac{1}{2 \Lambda}\left(\partial^{\theta} \bar{\Psi}^{C} \partial_{\theta} \Psi-M^{2} \bar{\Psi}^{C} \Psi+\text { H.c. }\right)\right] .
\end{aligned}
$$

This action implies that in the horizontal directions $\Psi$ behaves dynamically like a massless fermion but in the vertical direction it behaves like a "scalar" of the mass $M$.

Clearly, the vertical kinetic term $S_{V}[\Psi]$ in the action (37) is only allowed for the Weyl spinor fields which are neutral under any exact charge. On the other hand, if the field $\Psi$ transforms non-trivially under the local gauge symmetry such as $\mathrm{SU}(3)_{C} \times \mathrm{SU}(2)_{L} \times \mathrm{U}(1)_{Y}$ or carries out conserved charges such as electric charge, then this term $S_{V}[\Psi]$ should not be invariant and thus be forbidden. This means that such field $\Psi$ itself has no the term $S_{V}[\Psi]$ determining the $\theta$-dependence or the dynamics of $\Psi$ along the vertical direction in the spacetime $M_{5}$. By this, such field $\Psi$ itself must have a specific $\theta$-dependence which should be determined by a certain property. (Also, such field $\Psi$ has no Kaluza-Klein excitations in the effective four-dimensional spacetime.) This property is naturally that the field $\Psi$ is invariant under the active action of the Lie group U(1), which has been discussed in Sect. 2, meaning that

$\Psi\left(x, e^{i \theta}\right)=\frac{\psi(x)}{\sqrt{2 \pi R}} e^{i X_{\Psi} \theta}$,

where $\psi(x)$ is the corresponding Weyl spinor and identified as the usual four-dimensional fermion field. Note that, the factor $1 / \sqrt{2 \pi R}$ here will be used to normalize the fields in the effective four-dimensional spacetime. Under the general coordinate transformation (2), we have $\Psi^{\prime}\left(x^{\prime}, e^{i \theta^{\prime}}\right)=$ $\Psi\left(x, e^{i \theta}\right)$, thus the four-dimensional fermion field $\psi$ must transform as

$\psi(x) \longrightarrow \psi^{\prime}(x)=e^{-i X_{\Psi} \alpha(x)} \psi(x)$.

By substituting the expression (38) into the action (37) (with the term $S_{V}[\Psi]$ to be forbidden), one can derive the action for the fermion field $\psi(x)$ propagating dynamically in the effective four-dimensional spacetime, as

$S[\psi]=\int d^{4} x \bar{\psi} i \gamma^{\mu}\left(\partial_{\mu}-i g_{X} X_{\Psi} X_{\mu}\right) \psi$.

In this way, by starting from an action for the fermion field $\Psi$ [which is invariant under the active action of the Lie group $\mathrm{U}(1)]$ in the spacetime $M_{5}$, we derive an action for the fermion field $\psi$ respecting for the local gauge invariance $\mathrm{U}(1)_{X}$ in the effective four-dimensional spacetime. Here, the local gauge transformation of $\psi(x)$ under $\mathrm{U}(1)_{X}$ is understood as a result of the general transformation of $\Psi$ in the fiber coordinate of the five-dimensional spacetime $M_{5}$. And, the $\mathrm{U}(1)_{X}$ charge of $\psi$ is actually the quantum number which characterizes the active action of the Lie group U(1) on $\Psi$.

\section{A realistic model}

\subsection{Fermion and gauge sectors}

We begin by considering a set of the fermions fields, propagating dynamically in the spacetime $M_{5}$, which all respect for the gauge symmetry group $\mathrm{SU}(3)_{C} \otimes \mathrm{SU}(2)_{L} \otimes \mathrm{U}(1)_{Y}$. The fermion content under consideration is given as

$$
\begin{aligned}
& L_{a}\left(x, e^{i \theta}\right)=\left(\begin{array}{c}
N_{a L}\left(x, e^{i \theta}\right) \\
E_{a L}\left(x, e^{i \theta}\right)
\end{array}\right) \sim\left(1,2,-\frac{1}{2}\right), \\
& E_{a R}\left(x, e^{i \theta}\right) \sim(1,1,-1), \\
& N_{a R}\left(x, e^{i \theta}\right) \sim(1,1,0), \\
& Q_{a}\left(x, e^{i \theta}\right)=\left(\begin{array}{c}
U_{a L}\left(x, e^{i \theta}\right) \\
D_{a L}\left(x, e^{i \theta}\right)
\end{array}\right) \sim\left(3,2, \frac{1}{6}\right), \\
& D_{a R}\left(x, e^{i \theta}\right) \sim\left(3,1,-\frac{1}{3}\right), \\
& U_{a R}\left(x, e^{i \theta}\right) \sim\left(3,1, \frac{2}{3}\right),
\end{aligned}
$$

where quantum numbers in parentheses are to correspond to the gauge symmetries $\left\{\mathrm{SU}(3)_{C}, \mathrm{SU}(2)_{L}, \mathrm{U}(1)_{Y}\right\}$, respectively, and $a=1,2,3$ refer to three generations. Note that, the electric charge of the fields is defined as

$Q=T_{3}+Y$. 
Under the gauge symmetry group $\mathrm{SU}(3)_{C} \otimes \mathrm{SU}(2)_{L} \otimes \mathrm{U}(1)_{Y}$, the fermion fields transform as follows

$$
\begin{aligned}
\mathrm{SU}(3)_{C}: F \longrightarrow F^{\prime} & =e^{-i \alpha_{a} \frac{\lambda^{a}}{2}} F, \\
\mathrm{SU}(2)_{L}: F \longrightarrow F^{\prime} & =e^{-i \beta_{i} \frac{\sigma^{i}}{2}} F, \\
\mathrm{U}(1)_{Y}: F \longrightarrow F^{\prime} & =e^{-i \gamma} F,
\end{aligned}
$$

where the transforming parameters $\left\{\alpha_{a}, \beta_{i}, \gamma\right\}$ are the functions of the spacetime coordinates.

Action describing the propagation of the fermion in the spacetime $M_{5}$ is given by (37). As we mentioned, for the fermion fields which transform non-trivially under the gauge symmetry group $\mathrm{SU}(3)_{C} \otimes \mathrm{SU}(2)_{L} \otimes \mathrm{U}(1)_{Y}$, the vertical kinetic term $S_{V}[\Psi]$ in the action (37) should not be invariant and thus be forbidden, except the right-handed neutrinos $N_{a R}$. And, as a result, they themselves must have a specific $\theta$-dependence as

$$
\begin{gathered}
L_{a}\left(x, e^{i \theta}\right)=\frac{1}{\sqrt{2 \pi R}}\left(\begin{array}{c}
v_{a L}(x) \\
e_{a L}(x)
\end{array}\right) e^{i X_{L} \theta} \equiv \frac{l_{a}(x)}{\sqrt{2 \pi R}} e^{i X_{L} \theta}, \\
E_{a R}\left(x, e^{i \theta}\right)=\frac{e_{a R}(x)}{\sqrt{2 \pi R}} e^{i X_{E} \theta}, \\
Q_{a}\left(x, e^{i \theta}\right)=\frac{1}{\sqrt{2 \pi R}}\left(\begin{array}{c}
u_{a L}(x) \\
d_{a L}(x)
\end{array}\right) e^{i X_{Q} \theta} \equiv \frac{q_{a}(x)}{\sqrt{2 \pi R}} e^{i X_{Q} \theta}, \\
D_{a R}\left(x, e^{i \theta}\right)=\frac{d_{a R}(x)}{\sqrt{2 \pi R}} e^{i X_{D} \theta}, \\
U_{a R}\left(x, e^{i \theta}\right)=\frac{u_{a R}(x)}{\sqrt{2 \pi R}} e^{i X_{U} \theta},
\end{gathered}
$$

which all are invariant under the active action of the Lie group $\mathrm{U}(1)$, discussed in detail in Sect. 2. Here, the fields $l_{a}$, $e_{a R}, q_{a}, d_{a R}, u_{a R}$ are identified as the usual fermion fields in the SM. And, $\left(X_{L}, X_{E}, X_{Q}, X_{D}, X_{U}\right)$ are quantum numbers characterizing the active action of the Lie group $\mathrm{U}(1)$ on these fields. As indicated in appendix B, these quantum numbers are constrained by the anomaly cancellation of theory in the effective four-dimensional spacetime, given explicitly as

$$
\begin{aligned}
& X_{L}=-x, \quad X_{E}=-2 x, \quad X_{Q}=\frac{x}{3}, \\
& X_{D}=-\frac{2 x}{3}, \quad X_{D}=\frac{4 x}{3},
\end{aligned}
$$

where $x$ is a free parameter. From (44), it is easily to see that the transforming parameters in (43) are completely independent on the fiber coordinate $\theta$ but only dependent on the $x$-coordinates, meaning that

$$
\begin{aligned}
& \alpha_{a}=\alpha_{a}(x), \\
& \beta_{i}=\beta_{i}(x), \quad \gamma=\gamma(x) .
\end{aligned}
$$

This suggests clearly the simplest form for the gauge fields corresponding to $\left\{\mathrm{SU}(3)_{C}, \mathrm{SU}(2)_{L}, \mathrm{U}(1)_{Y}\right\}$ as

$$
\begin{aligned}
G_{a M} & =\left(\frac{G_{a \mu}(x)}{\sqrt{2 \pi R}}, 0\right), \\
W_{i M} & =\left(\frac{W_{i \mu}(x)}{\sqrt{2 \pi R}}, 0\right), \\
B_{M} & =\left(\frac{B_{\mu}(x)}{\sqrt{2 \pi R}}, 0\right),
\end{aligned}
$$

which are only dependent on the $x$-coordinates and have all the zero vertical component.

We now can write bulk action for the gauge boson and fermion fields, up to the gauge fixing and ghost terms, as

$$
\begin{aligned}
S_{\mathrm{FG}}^{\text {bulk }}= & \int d x^{4} d \theta \sqrt{|\operatorname{det} G|}\left(\mathcal{L}_{\text {gauge }}^{\text {bulk }}+\mathcal{L}_{\mathrm{fer}}^{\text {bulk }}\right), \\
\mathcal{L}_{\text {gauge }}^{\text {bulk }}= & -\frac{1}{4} G_{a M N} G_{a}^{M N} \\
& -\frac{1}{4} W_{i M N} W_{i}^{M N}-\frac{1}{4} B_{M N} B^{M N}+\frac{M_{*}^{3}}{2} \mathcal{R}, \\
\mathcal{L}_{\text {fer }}^{\text {bulk }}= & \sum_{F} \bar{F} i \gamma^{\mu} \hat{D}_{\mu} F+\bar{N}_{a R} i \gamma^{\mu} \hat{\partial}_{\mu} N_{a R} \\
& +\frac{1}{2 \Lambda}\left(\partial^{\theta} \bar{N}_{a R}^{C} \partial_{\theta} N_{a R}-M_{N_{a}}^{2} \bar{N}_{a R}^{C} N_{a R}+\text { H.c. }\right),
\end{aligned}
$$

where $\left\{G_{a M N}, W_{i M N}, B_{M N}\right\}$ are the field strength tensors of the gauge fields $\left\{G_{a M}, W_{i M}, B_{M}\right\}$, which have the non-zero components given by (up to a normalized factor)

$$
\begin{aligned}
& G_{a \mu \nu}=\partial_{\mu} G_{a \nu}-\partial_{\nu} G_{a \mu}+g_{s} f_{a b c} A_{b \mu} A_{c \nu} \\
& W_{i \mu \nu}=\partial_{\mu} W_{i \nu}-\partial_{\nu} W_{i \mu}+g \varepsilon_{i j k} W_{j \mu} W_{k \nu} \\
& B_{\mu \nu}=\partial_{\mu} B_{\nu}-\partial_{\nu} B_{\mu}
\end{aligned}
$$

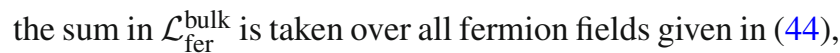
$M_{N_{a}}$ are the vertical mass parameters of the right-handed neutrinos $N_{a R}$ which are naturally the order of the scale $\Lambda$, and the covariant derivative $\hat{D}_{\mu}$ reads

$\hat{D}_{\mu}=\hat{\partial}_{\mu}-i g_{s} \frac{\lambda^{a}}{2} G_{a \mu}-i g \frac{\sigma^{i}}{2} W_{i \mu}-i g^{\prime} Y_{F} B_{\mu}$,

with $\left\{g_{s}, g, g^{\prime}\right\}$ to be coupling constants of the gauge symmetries $\left\{\mathrm{SU}(3)_{C}, \mathrm{SU}(2)_{L}, \mathrm{U}(1)_{Y}\right\}$. Note that, as we indicated in the previous section, the last term in $\mathcal{L}_{\text {gauge }}^{\text {bulk }}$ defines Lagrangian for the gauge field $X_{\mu}$. Then, we can obtain action 
in the effective four-dimensional spacetime as

$$
\begin{aligned}
S_{\mathrm{FG}}^{\mathrm{eff}}=\int & d x^{4}\left(-\frac{1}{4} G_{a \mu \nu} G^{a \mu \nu}-\frac{1}{4} W_{i \mu \nu} W^{i \mu \nu}\right. \\
& -\frac{1}{4} B_{\mu \nu} B^{\mu \nu}-\frac{1}{4} X_{\mu \nu} X^{\mu \nu} \\
& \left.+\sum_{f} \bar{f} i \gamma^{\mu} D_{\mu} f+\mathcal{L}_{N}\right)
\end{aligned}
$$

where the effective fermion $f(x)$ is related to the fundamental fermion $F\left(x, e^{i \theta}\right)$ by

$F\left(x, e^{i \theta}\right)=\frac{f(x)}{\sqrt{2 \pi R}} e^{i X_{F} \theta}$,

the covariant derivative $D_{\mu}$ reads

$D_{\mu}=\partial_{\mu}-i g_{s} \frac{\lambda^{a}}{2} G_{a \mu}-i g \frac{\sigma^{i}}{2} W_{i \mu}-i g^{\prime} Y_{f} B_{\mu}-i g_{X} X_{f} X_{\mu}$,

(with $Y_{F}$ and $X_{F}$ to be replaced by $Y_{f}$ and $X_{f}$, respectively, for a convenient reason) and $\mathcal{L}_{N}$ is an effective Lagrangian corresponding to the right-handed neutrinos $N_{a R}$. We make several remarks here. First, the action (51) looks like an extension of the SM based on the gauge symmetry group $\mathrm{SU}(3)_{C} \otimes \mathrm{SU}(2)_{L} \otimes \mathrm{U}(1)_{Y} \otimes \mathrm{U}(1)_{X}$, where the $\mathrm{U}(1)_{X}$ charges of the SM fermions are given explicitly in Table 1.

But, there are remarkable differences compared to the usual U(1) extensions of the SM, which we will see below. Second, the fermions and the gauge bosons of the SM have no the Kaluza-Klein (KK) counterparts. This is due to the fact that the vertical kinetic term $S_{V}[\Psi]$ of the corresponding bulk fermions is forbidden because they transform non-trivially under the gauge symmetry group $\mathrm{SU}(3)_{C} \otimes \mathrm{SU}(2)_{L} \otimes \mathrm{U}(1)_{Y}$.

In order to find the effective action $\mathcal{L}_{N}$ for the right-handed neutrinos $N_{a R}$ in the effective four-dimensional spacetime, we need to perform its KK decomposition along the vertical direction of the spacetime $M_{5}$. First, let us obtain (free) equation of motion from the action for $N_{a R}$ in (48) as

$i \gamma^{\mu} \partial_{\mu} N_{a R}-\frac{1}{\Lambda} \partial_{\theta} \partial^{\theta} N_{a R}^{C}-\frac{M_{N_{a}}^{2}}{\Lambda} N_{a R}^{C}=0$

where $\partial_{\theta} \partial^{\theta}=-\frac{1}{R^{2}} \frac{\partial^{2}}{\partial \theta^{2}}$. This equation is solved by the variable separation $N_{a R}=A(x) Y(\theta)$, where $Y(\theta)$ satisfies the periodic condition $Y(\theta)=Y(\theta+2 \pi)$. Then, we can easily obtain the equation for $Y(\theta)$ as, $d^{2} Y(\theta) / d \theta^{2}=-n^{2}$, which leads to

$Y(\theta)=\cos (n \theta)$ or $Y(\theta)=\sin (n \theta)$,

where $n=0,1,2,3, \ldots$ are non-negative integer numbers. Since we can expand the right-handed neutrinos $N_{a R}$ in terms of these functions as

$$
\begin{aligned}
N_{a R}\left(x, e^{i \theta}\right)= & \frac{v_{a R}(x)}{\sqrt{2 \pi R}} \\
& +\frac{1}{\sqrt{\pi R}} \sum_{n=1}^{\infty}\left[\psi_{n a R} \cos (n \theta)+\chi_{n a R} \sin (n \theta)\right],
\end{aligned}
$$

where $v_{a R}(x)$ are identified as the usual right-handed neutrinos, and $\left\{\psi_{n a R}, \chi_{n a R}\right\}$ are their KK excitations. Note that, $\psi_{n a R}$ and $\chi_{n a R}$ are different types of the KK excitations which correspond to the partial solutions $\cos (n \theta)$ and $\sin (n \theta)$, respectively. By substituting this KK expansion into the action for $N_{a R}$ in (48), we obtain the effective action $\mathcal{L}_{N}$ as

$$
\begin{aligned}
\mathcal{L}_{N} & =\mathcal{L}_{v}+\mathcal{L}_{\psi}+\mathcal{L}_{\chi}+\mathcal{L}_{\mathrm{int}}, \\
\mathcal{L}_{v} & =\bar{v}_{a R} i \gamma^{\mu} \partial_{\mu} v_{a R}-\frac{M_{a 0}}{2} \bar{v}_{a R}^{C} v_{a R}+\text { H.c, } \\
\mathcal{L}_{\psi} & =\sum_{n=1}^{\infty}\left(\bar{\psi}_{n a R} i \gamma^{\mu} \partial_{\mu} \psi_{n a R}-\frac{M_{a n}}{2} \bar{\psi}_{n a R}^{C} \psi_{n a R}+\text { H.c. }\right), \\
\mathcal{L}_{\chi}= & \sum_{n=1}^{\infty}\left(\bar{\chi}_{n a R} i \gamma^{\mu} \partial_{\mu} \chi_{n a R}-\frac{M_{a n}}{2} \bar{\chi}_{n a R}^{C} \chi_{n a R}+\text { H.c. }\right), \\
\mathcal{L}_{\text {int }}= & i g_{X} \sum_{n=1}^{\infty} n\left(\bar{\chi}_{n a R} \gamma^{\mu} \psi_{n a R}-\bar{\psi}_{n a R} \gamma^{\mu} \chi_{n a R}\right) X_{\mu},
\end{aligned}
$$

where

$$
\begin{aligned}
& M_{a 0}=\frac{M_{N_{a}}^{2} \sim \Lambda,}{\Lambda} \sim \\
& M_{a n}=\frac{1}{\Lambda}\left(M_{N_{a}}^{2}+\frac{n^{2}}{R^{2}}\right) \sim \Lambda .
\end{aligned}
$$

From the action $\mathcal{L}_{N}$, first the $\mathrm{U}(1)_{X}$ charges of the usual right-handed neutrinos $v_{a R}$ are all zero. This is different to the usual $\mathrm{U}(1)$ extensions of the SM, such as the $\mathrm{U}(1)_{B-L}$ extension. [In the usual U(1) extensions of the SM, the righthanded neutrinos need to carry the non-zero charges under the additional U(1). This is because the right-handed neutrinos should acquire the heavy Majorana masses after the spontaneous breaking of this symmetry group.] Second, the usual right-handed neutrinos $v_{a R}$ and their KK excitations

Table 1 The U(1) $X$ charges of the fermions. Note that, $X_{v_{a L}}=X_{e_{a L}}=X_{L}, X_{e_{a R}}=X_{E}, X_{u_{a L}}=X_{d_{a L}}=X_{Q}, X_{u_{a R}}=X_{U}$, and $X_{d_{a R}}=X_{D}$

\begin{tabular}{llllllll}
\hline Fermion $f$ & $v_{a L}$ & $e_{a L}$ & $e_{a R}$ & $u_{a L}$ & $d_{a L}$ & $u_{a R}$ & $d_{a R}$ \\
\hline$X_{f}$ & $-x$ & $-x$ & $-2 x$ & $\frac{1}{3} x$ & $\frac{1}{3} x$ & $\frac{4}{3} x$ & $-\frac{2}{3} x$ \\
\hline
\end{tabular}


possess naturally the heavy Majorana masses which are generated by the kinetic term describing the propagation of the corresponding bulk neutrinos $N_{a R}$ along the vertical direction in the spacetime $M_{5}$. Third, the couplings of the gauge boson $X$ to the KK excitations of $v_{a R}$ are unusual. More specifically, the gauge boson $X$ couples to a KK excitation and KK anti-excitation of another field.

\subsection{Scalar sector and symmetry breaking}

Now we turn to the scalar sector in this model, which includes a doublet scalar field $H$ and a singlet complex scalar field $\Phi$, as

$$
\begin{gathered}
H\left(x, e^{i \theta}\right)=\left(\begin{array}{c}
\phi^{+}\left(x, e^{i \theta}\right) \\
\phi^{0}\left(x, e^{i \theta}\right)
\end{array}\right) \sim\left(1,2, \frac{1}{2}\right), \\
\Phi\left(x, e^{i \theta}\right) \sim(1,1,0),
\end{gathered}
$$

Here, we assume that $\Phi$ is invariant under the active action of the Lie group U(1) and thus given as

$\Phi\left(x, e^{i \theta}\right)=\frac{\varphi(x)}{\sqrt{2 \pi R}} e^{i X_{\Phi} \theta}$.

For simplicity, in this paper we set $X_{\Phi}=1$. (If $X_{\Phi} \neq 1$, the related constraints will correspond to the gauge coupling by scaling as $g_{X} / X_{\Phi}$.) Action, describing the dynamical propagation of these scalar fields in the spacetime $M_{5}$, is given by

$$
\begin{aligned}
S[H, \Phi]=\int & d^{4} x d \theta \sqrt{|\operatorname{det} G|}\left[\left|\left(\hat{\partial}_{\mu}-i g \frac{\sigma^{i}}{2} W_{i \mu}-i \frac{g^{\prime}}{2} B_{\mu}\right) H\right|^{2}\right. \\
& \left.+\left|\partial_{\theta} H\right|^{2}+\left|\hat{\partial}_{\mu} \Phi\right|^{2}-V(H, \Phi)\right],
\end{aligned}
$$

where the scalar potential, invariant under the gauge symmetries, is given by

$$
\begin{gathered}
V(H, \Phi)=\mu_{1}^{2} H^{\dagger} H+\bar{\lambda}_{1}\left(H^{\dagger} H\right)^{2}+\mu_{2}^{2} \Phi^{\dagger} \Phi+\bar{\lambda}_{2}\left(\Phi^{\dagger} \Phi\right)^{2} \\
+\bar{\lambda}_{3}\left(H^{\dagger} H\right)\left(\Phi^{\dagger} \Phi\right) .
\end{gathered}
$$

Note that, because the scalar field $\Phi$ has a specific $\theta$ dependence given at (60), its vertical kinetic term leads to a mass term which is absorbed into the potential $V(H, \Phi)$. In order to find the effective action $S^{\text {eff }}$ for the scalar sector in the effective four-dimensional spacetime, let first us perform the KK decomposition for the scalar field $H$ as

$$
\begin{aligned}
H\left(x, e^{i \theta}\right)= & \frac{\phi(x)}{\sqrt{2 \pi R}} \\
& \quad+\frac{1}{\sqrt{\pi R}} \sum_{n=1}^{\infty}\left[\phi_{1 n} \cos (n \theta)+\phi_{2 n} \sin (n \theta)\right],
\end{aligned}
$$

where $\phi(x)$ is identified as the SM scalar doublet and $\left\{\phi_{1 n}, \phi_{2 n}\right\}$ are its KK counterparts. Then, the effective action $S^{\text {eff }}$ reads

$$
\begin{aligned}
& S^{\mathrm{eff}}=\int d^{4} x\left\{\mathcal{L}[\phi, \varphi]+\mathcal{L}\left[\phi_{1 n}, \phi_{2 n}\right]+\cdots\right\} \\
& \mathcal{L}[\phi, \varphi]=\left|D_{\mu} \phi\right|^{2}+\left|\left(\partial_{\mu}-i g_{X} X_{\mu}\right) \varphi\right|^{2}-V(\phi, \varphi), \\
& \mathcal{L}\left[\phi_{1 n}, \phi_{2 n}\right]=\left|D_{\mu} \phi_{1 n}\right|^{2}+\left|D_{\mu} \phi_{2 n}\right|^{2} \\
& \quad+g_{X} \sum_{n=1}^{\infty}\left[i n \phi_{2 n}^{\dagger} D_{\mu} \phi_{1 n} X^{\mu}+i n\left(D_{\mu} \phi_{2 n}\right)^{\dagger} \phi_{1 n} X^{\mu}+\text { H.c. }\right] \\
& \quad+g_{X}^{2} \sum_{n=1}^{\infty} n^{2}\left(\phi_{1 n}^{\dagger} \phi_{1 n}+\phi_{2 n}^{\dagger} \phi_{2 n}\right) X_{\mu} X^{\mu} \\
& \quad+\mu_{n}^{2}\left(\phi_{1 n}^{\dagger} \phi_{1 n}+\phi_{2 n}^{\dagger} \phi_{2 n}\right),
\end{aligned}
$$

where $D_{\mu}=\partial_{\mu}-i g \frac{\sigma^{i}}{2} W_{i \mu}-i \frac{g^{\prime}}{2} B_{\mu}$, the potential $V(\phi, \varphi)$ is

$$
\begin{aligned}
V(\phi, \varphi)= & \mu_{1}^{2} \phi^{\dagger} \phi+\lambda_{2}\left(\phi^{\dagger} \phi\right)^{2} \\
& +\mu_{2}^{2} \varphi^{\dagger} \varphi+\lambda_{2}\left(\varphi^{\dagger} \varphi\right)^{2}+\lambda_{3}\left(\phi^{\dagger} \phi\right)\left(\varphi^{\dagger} \varphi\right) .
\end{aligned}
$$

with $\mu_{\underline{n}}^{2}=\mu_{1}^{2}+n^{2} / R^{2}, \lambda_{1}=\bar{\lambda}_{1} / 2 \pi R, \lambda_{2}=\bar{\lambda}_{2} / 2 \pi R$, $\lambda_{3}=\bar{\lambda}_{3} / 2 \pi R$, and the ellipse refers to the self-coupling terms of the KK excitations $\left\{\phi_{1 n}, \phi_{2 n}\right\}$ and their couplings to $\phi$ and $\varphi$ which are irrelevant in the following discussions. Note that, the $\mathrm{U}(1)_{X}$ charge of the SM scalar doublet $\phi$ is zero and thus it does not couple directly to the gauge boson $X$.

For negative squared masses, $\mu_{1}^{2}<0$ and $\mu_{2}^{2}<0$, the scalar fields $\phi$ and $\varphi$ should develop non-vanishing vacuum expectation values (VEVs). However, because the energy scale $\Lambda$ is much more than the electroweak scale, the squared masses $\mu_{n}^{2}$ of the KK excitations $\phi_{1 n}$ and $\phi_{2 n}$ remain positive. As a result, their VEV is zero. In this way, we have

$$
\begin{aligned}
\langle\phi\rangle & =\frac{1}{\sqrt{2}}\left(\begin{array}{l}
0 \\
v
\end{array}\right), \\
\langle\varphi\rangle & =\frac{v^{\prime}}{\sqrt{2}}, \\
\left\langle\phi_{1 n}\right\rangle & =\left\langle\phi_{2 n}\right\rangle=0,
\end{aligned}
$$

where

$$
\begin{aligned}
v^{2} & =2 \frac{2 \lambda_{2} \mu_{1}^{2}-\lambda_{3} \mu_{2}^{2}}{\lambda_{3}^{2}-4 \lambda_{1} \lambda_{2}}, \\
v^{\prime 2} & =2 \frac{2 \lambda_{1} \mu_{2}^{2}-\lambda_{3} \mu_{1}^{2}}{\lambda_{3}^{2}-4 \lambda_{1} \lambda_{2}} .
\end{aligned}
$$

For the potential bounded from below, the following conditions must be satisfied, $\lambda_{3}>2 \sqrt{\lambda_{1} \lambda_{2}}$ and $\lambda_{1,2} \geq 0$. Once $\varphi$ develops the nonzero $\mathrm{VEV}$, the gauge boson $X_{\mu}$ acquires a mass, $M_{X}=g_{X} v^{\prime}$. Using the relation (32), the mass of the gauge boson $X$ is expressed as

$$
M_{X}=\frac{\sqrt{2} \Lambda v^{\prime}}{T_{0} M_{P l}}<\frac{\Lambda}{T_{0}},
$$


which implies that the gauge boson $X$ is lighter than all KK excitations. After the symmetry breaking, the KK excitations $\left\{\phi_{1 n}, \phi_{2 n}\right\}$ are still the physical fields of the mass $\mu_{n}$. Whereas, the CP-even excitations of $\phi$ and $\varphi$, which are not eaten by the gauge bosons $\left\{W^{ \pm}, Z_{\mu}, X_{\mu}\right\}$, should mix together. We expand these scalar fields in the unitary gauge around the vacuum as

$\phi=\frac{1}{\sqrt{2}}\left(\begin{array}{c}0 \\ v+h(x)\end{array}\right), \quad \varphi=\frac{v^{\prime}+h^{\prime}(x)}{\sqrt{2}}$.

Their mass term is given by

$\mathcal{L}_{\text {mass }}\left(h, h^{\prime}\right)=\frac{1}{2}\left(h h^{\prime}\right)\left(\begin{array}{ccc}2 \lambda_{1} v^{2} & \lambda_{3} v v^{\prime} \\ \lambda_{3} v v^{\prime} & 2 \lambda_{2} v^{\prime 2}\end{array}\right)\left(\begin{array}{c}h \\ h^{\prime}\end{array}\right)$.

The physical states are found as, $h_{1}=c_{\alpha} h+s_{\alpha} h^{\prime}$ and $h_{2}=$ $-s_{\alpha} h+c_{\alpha} h^{\prime}$, corresponding to the following masses

$m_{h_{1}, h_{2}}^{2}=\lambda_{2} v^{2}+\lambda_{1} v^{\prime} \mp\left[\left(\lambda_{2} v^{2}-\lambda_{1} v^{\prime}\right)^{2}+\lambda_{3}^{2} v^{2} v^{\prime 2}\right]^{1 / 2}$.

The lighter state $h_{1}$ is identified as the SM Higgs discovered at LHC, and thus $m_{h_{1}}=125 \mathrm{GeV}$, whereas the heavier one $h_{2}$ has so far been escaped the detections. The mixing angle is defined as, $\sin 2 \alpha=2 \lambda_{3} v v^{\prime} /\left(m_{h_{2}}^{2}-m_{h_{1}}^{2}\right)$, which is constrained by the data from the measurements of the Higgs production cross section and its decay branching ratio at the LHC [69]. If $v \ll v^{\prime}$, with $\lambda_{1} \sim \lambda_{2} \sim \lambda_{3}$, we have $\sin \alpha \simeq \frac{2 v}{v^{\prime}} \ll 1$ which implies that the mixing effects can be neglected. Interestingly, the mixing between $h$ and $h^{\prime}$ leads to the presence of the direct couplings between the gauge boson $X_{\mu}$ with the SM Higgs $h_{1}$, although the SM scalar doublet $\phi$ does not carry the charge under the group $\mathrm{U}(1)_{X}$. The coupling of the exotic Higgs $h_{2}$ to the SM particles generically goes through the gauge boson $X$ and the mixing between it and the SM Higgs.

\subsection{Mass spectrum for fermions}

The Yukawa couplings between the fermions and the scalar double $H$ are given by the bulk Yukawa action

$$
\begin{aligned}
S_{\text {Yukawa }}^{\text {bulk }}= & \int d^{4} x d \theta \sqrt{|\operatorname{det} G|} \\
& \times\left(\bar{h}_{a b}^{e} \bar{L}_{a} H E_{b R}+\bar{h}_{a b}^{v} \bar{L}_{a} \widetilde{H} N_{b R}+\bar{h}_{a b}^{d} \bar{Q}_{a} H D_{b R}\right. \\
& \left.+\bar{h}_{a b}^{u} \bar{Q}_{a} \tilde{H} U_{b R}+\text { H.c. }\right)
\end{aligned}
$$

where $\widetilde{H}=i \sigma^{2} H^{*}$. After spontaneous symmetry breaking, we find the Dirac mass terms in the effective four- dimensional spacetime for the fermions as

$$
\begin{aligned}
S_{\text {mass }}=- & \int d^{4} x\left[m_{a b}^{e} \bar{e}_{a L} e_{b R}+m_{a b}^{v} \bar{v}_{a L} v_{b R}\right. \\
& +\sum_{n=1}^{\infty}\left(m_{n a b}^{\psi} \bar{v}_{a L} \psi_{n b R}+m_{n a b}^{\chi} \bar{v}_{a L} \chi_{n b R}\right)+ \\
& \left.+m_{a b}^{d} \bar{d}_{a L} d_{b R}+m_{a b}^{u} \bar{u}_{a L} u_{b R}+\text { H.c. }\right]
\end{aligned}
$$

where the mass matrices read

$$
\begin{aligned}
& m_{a b}^{e}=\frac{i\left(1-e^{-2 \pi i x}\right) \bar{h}_{a b}^{e}}{4 \pi x \sqrt{\pi R}} v \equiv h_{a b}^{e} \frac{v}{\sqrt{2}}, \\
& m_{a b}^{v}=\frac{i\left(e^{2 \pi i x}-1\right) \bar{h}_{a b}^{v}}{4 \pi x \sqrt{\pi R}} v \equiv h_{a b}^{v} \frac{v}{\sqrt{2}}, \\
& m_{a b}^{d}=\frac{i\left(1-e^{-2 \pi i x}\right) \bar{h}_{a b}^{d}}{4 \pi x \sqrt{\pi R}} v \equiv h_{a b}^{d} \frac{v}{\sqrt{2}}, \\
& m_{a b}^{u}=\frac{i\left(e^{2 \pi i x}-1\right) \bar{h}_{a b}^{u}}{4 \pi x \sqrt{\pi R}} v \equiv h_{a b}^{u} \frac{v}{\sqrt{2}}, \\
& m_{n a b}^{\psi}=\frac{i x\left(1-e^{2 \pi i x}\right) \bar{h}_{a b}^{v}}{2 \pi \sqrt{2 \pi R}\left(n^{2}-x^{2}\right)} v \equiv h_{n a b}^{\psi} \frac{v}{\sqrt{2}}, \\
& m_{n a b}^{\chi}=\frac{n\left(e^{2 \pi i x}-1\right) \bar{h}_{a b}^{v}}{2 \pi \sqrt{2 \pi R}\left(n^{2}-x^{2}\right)} v \equiv h_{n a b}^{\chi} \frac{v}{\sqrt{2}} .
\end{aligned}
$$

The fact that the mass matrices of the SM fermions are nonzero requires the following condition

$$
\begin{gathered}
1-e^{-2 \pi i x} \neq 0 \quad \& e^{2 \pi i x}-1 \neq 0 \\
\Longrightarrow x \neq \pm 1, \pm 2, \pm 3, \ldots,
\end{gathered}
$$

which restricts the possible values of the free parameter $x$. It is important to note here that the Dirac masses $\left\{m_{n a b}^{\psi}, m_{n a b}^{\chi}\right\}$ should decrease in increasing of the KK excitation order $n$. On the other hand, $m_{n a b}^{\psi}$ and $m_{n a b}^{\chi}$ should approach zero as $n \rightarrow \infty .\left\{h_{a b}^{e}, h_{a b}^{u}, h_{a b}^{d}\right\}$ are identified as the usual Yukawa coupling constants in the SM, thus the masses of the fermions in both charged-lepton and quark sector are the same as the SM one.

Now we are interested in the masses of the neutral fermions. By combining the Majorana mass terms in (57) and the Dirac mass terms in (73), we can find the mass Lagrangian for the neutral fermions as

$$
\begin{aligned}
\mathcal{L}_{\text {mass }}^{N}=- & \frac{1}{2}\left(\bar{v}_{L}, \bar{v}_{R}^{C}, \bar{\psi}_{1 R}^{C}, \bar{\chi}_{1 R}^{C}, \bar{\psi}_{2 R}^{C}, \bar{\chi}_{2 R}^{C}, \ldots\right) \\
& \times \mathcal{M}_{N}\left(\begin{array}{c}
v_{L}^{C} \\
v_{R} \\
\psi_{1 R} \\
\chi_{1 R} \\
\psi_{2 R} \\
\chi_{2 R} \\
\vdots
\end{array}\right)+\text { H.c. }
\end{aligned}
$$


where the block mass matrix $\mathcal{M}_{N}$ is given as

$$
\mathcal{M}_{N}=\left(\begin{array}{cccccccc}
0 & m^{\nu} & m_{1}^{\psi} & m_{1}^{\chi} & m_{2}^{\psi} & m_{2}^{\chi} & \cdots \\
\left(m^{\nu}\right)^{T} & M_{0} & 0 & 0 & 0 & 0 & \cdots \\
\left(m_{1}^{\psi}\right)^{T} & 0 & M_{1} & 0 & 0 & 0 & \cdots \\
\left(m_{1}^{\chi}\right)^{T} & 0 & 0 & M_{1} & 0 & 0 & \cdots \\
\left(m_{2}^{\psi}\right)^{T} & 0 & 0 & 0 & M_{2} & 0 & \cdots \\
\left(m_{2}^{\chi}\right)^{T} & 0 & 0 & 0 & 0 & M_{2} & \cdots \\
\vdots & \vdots & \vdots & \vdots & \vdots & \vdots & \ddots
\end{array}\right) .
$$

The diagonalization of this block mass matrix yields the following block mass eigenvalues

$$
\begin{aligned}
m^{\nu_{L}^{\prime}} \approx- & m^{v} M_{0}^{-1}\left(m^{\nu}\right)^{T} \\
& -\sum_{n=1}^{\infty}\left[m_{n}^{\psi} M_{n}^{-1}\left(m_{n}^{\psi}\right)^{T}+m_{n}^{\chi} M_{n}^{-1}\left(m_{n}^{\chi}\right)^{T}\right] \\
& \sim \frac{\left(h^{v} v\right)^{2}}{\Lambda}, \\
m^{v_{R}^{\prime}} \approx M_{0} & \sim \Lambda, \quad m_{n}^{\psi^{\prime}} \approx M_{n} \sim \Lambda, \quad m_{n}^{\chi^{\prime}} \approx M_{n} \sim \Lambda .
\end{aligned}
$$

Note that, the contributions from the KK excitations $\psi_{n R}$ and $\chi_{n R}$ in $m^{\nu_{L}^{\prime}}$ decrease approximately in $\frac{1}{n^{6}}$ and $\frac{1}{n^{4}}$, respectively. Thus, with respect to the high-order KK excitations, their contributions in $m^{\nu_{L}^{\prime}}$ can be negligible. The corresponding eigenvectors $\left(v_{L}^{\prime C}, \psi_{1 R}^{\prime}, \chi_{1 R}^{\prime}, \psi_{2 R}^{\prime}, \chi_{2 R}^{\prime}, \ldots\right)^{T}$ are determined by

$$
\left(\begin{array}{c}
\nu_{L}^{\prime C} \\
v_{R}^{\prime} \\
\psi_{1 R}^{\prime} \\
\chi_{1 R}^{\prime} \\
\psi_{2 R}^{\prime} \\
\chi_{2 R}^{\prime} \\
\vdots
\end{array}\right) \approx\left(\begin{array}{ccccccc}
1 & \delta^{\nu} & \delta_{1}^{\psi} & \delta_{1}^{\chi} & \delta_{2}^{\psi} & \delta_{2}^{\chi} & \cdots \\
-\left(\delta^{\nu}\right)^{T} & 1 & 0 & 0 & 0 & 0 & \cdots \\
-\left(\delta_{1}^{\psi}\right)^{T} & 0 & 1 & 0 & 0 & 0 & \cdots \\
-\left(\delta_{1}^{\chi}\right)^{T} & 0 & 0 & 1 & 0 & 0 & \ldots \\
-\left(\delta_{2}^{\psi}\right)^{T} & 0 & 0 & 0 & 1 & 0 & \ldots \\
-\left(\delta_{2}^{\chi}\right)^{T} & 0 & 0 & 0 & 0 & 1 & \ldots \\
\vdots & \vdots & \vdots & \vdots & \vdots & \vdots & \ddots
\end{array}\right)\left(\begin{array}{c}
v_{L}^{C} \\
v_{R} \\
\psi_{1 R} \\
\chi_{1 R} \\
\psi_{2 R} \\
\chi_{2 R} \\
\vdots
\end{array}\right),
$$

where $\delta^{\nu}=m^{\nu} M_{0}^{-1}, \delta_{n}^{\psi}=m_{n}^{\psi} M_{n}^{-1}$, and $\delta_{n}^{\chi}=m_{n}^{\chi} M_{n}^{-1}$. The physical states $U_{M N S} v_{L}^{\prime}$ (or the active neutrinos) and the mass eigenvalues $U_{M N S} m^{\nu_{L}^{\prime}} U_{M N S}^{T}$, where $U_{M N S}$ is the socalled Maki-Nakagawa-Sakata (MNS) matrix, are related the current neutrino oscillation data [69]. For the Yukawa coupling $h^{v} \sim \mathcal{O}\left(10^{-4}\right)$ which is the order of the Yukawa coupling of the muon, $\Lambda / T_{0}$ about $\sim 10^{3} \mathrm{TeV}$ can lead to the neutrino masses at the observed sub-eV scale. Whereas, with $h^{v} \sim \mathcal{O}(1)$ which is the order of the Yukawa coupling of the top quark, in order to produce the sub-eV neutrino mass scale, $\Lambda / T_{0}$ is about $\sim 10^{11} \mathrm{TeV}$. In this way, the fact that the neutrino masses are observed to be small is an unavoidable consequence in this scenario.

\subsection{Phenomenology of the gauge boson $X$}

The gauge boson $X$ has the couplings with quarks and leptons. Thus, it may be produced in (future) colliders, then it would decay to pairs of fermions. Now we study the treelevel decays of the gauge boson $X$ into the two-body final states. (The loop induced decays of the gauge boson $X$ will be studied in our future work.) Because there has no treelevel mixing between the gauge boson $X$ and the SM gauge bosons, $X$ should not decay into SM diboson pairs and the pairs $Z S$ with $S$ referring to any scalar. Also, the gauge boson $X$ can not decay into the pairs of $\left(\bar{\chi}_{n R}, \psi_{n R}\right)$ and $\left(\bar{\psi}_{n R}, \chi_{n R}\right)$, because it is lighter than these KK excitations. On the other hand, in this scenario, the gauge boson $X$ may only decay into the SM fermion pairs at the tree level. The couplings of the gauge boson $X$ to the fermion $f$ is given as

$\mathcal{L}_{\bar{f} f X}=\left(\lambda_{f_{L}} \bar{f}_{L} \gamma^{\mu} f_{L}+\lambda_{f_{R}} \bar{f}_{R} \gamma^{\mu} f_{R}\right) X_{\mu}$,

where $\lambda_{f_{L}}=g_{X} X_{f_{L}}$ and $\lambda_{f_{R}}=g_{X} X_{f_{R}}$ refer to the coupling factors of the gauge boson $X$ to the left and right handed fermions $f_{L}$ and $f_{R}$, respectively. Note that, with respect to the neutrinos, we have $\lambda_{v_{R}}=0$. In this scenario, because of $\lambda_{f_{L}} \neq \lambda_{f_{R}}$, the couplings of the gauge boson $X$ to the lefthanded and right-handed fermions are different. The partial decay width of the decay $X \rightarrow \bar{f} f$ is given by

$$
\begin{aligned}
& \Gamma(X \rightarrow \bar{f} f)=\frac{N_{C}(f) M_{X}}{24 \pi} \sqrt{1-\frac{4 m_{f}^{2}}{M_{X}^{2}}} \\
& \quad \times\left[\left(\lambda_{f L}^{2}+\lambda_{f R}^{2}\right)\left(1-\frac{m_{f}^{2}}{M_{X}^{2}}\right)+6 \lambda_{f L} \lambda_{f R} \frac{m_{f}^{2}}{M_{X}^{2}}\right],
\end{aligned}
$$

where $N_{C}(f)$ and $m_{f}$ ar the color number and the mass of the fermion $f$, respectively. The invisible decay width of the gauge boson $X$, which corresponds to its decay into neutrino and anti-neutrino pairs, is given

$$
\sum_{\nu=v_{e, \mu, \tau}} \Gamma(X \rightarrow \bar{v} \nu) \simeq \frac{g_{X}^{2} x^{2}}{8 \pi} M_{X} .
$$

We plot the branching ratios of the gauge boson $X$ as the functions of its mass $M_{X}$, in Fig. 2, which show

$$
\begin{aligned}
& \sum_{\nu=v_{e, \mu, \tau}} \operatorname{BR}(X \rightarrow \bar{v} v) \sim 7.5 \%, \\
& \sum_{l=e, \mu, \tau} \operatorname{BR}(X \rightarrow \bar{l} l) \sim 37.5 \%, \\
& \sum_{q=u, d, s, c, b} \operatorname{BR}(X \rightarrow \bar{q} q) \sim 40.8 \%, \\
& \operatorname{BR}(X \rightarrow \bar{t} t) \sim 14.2 \% .
\end{aligned}
$$




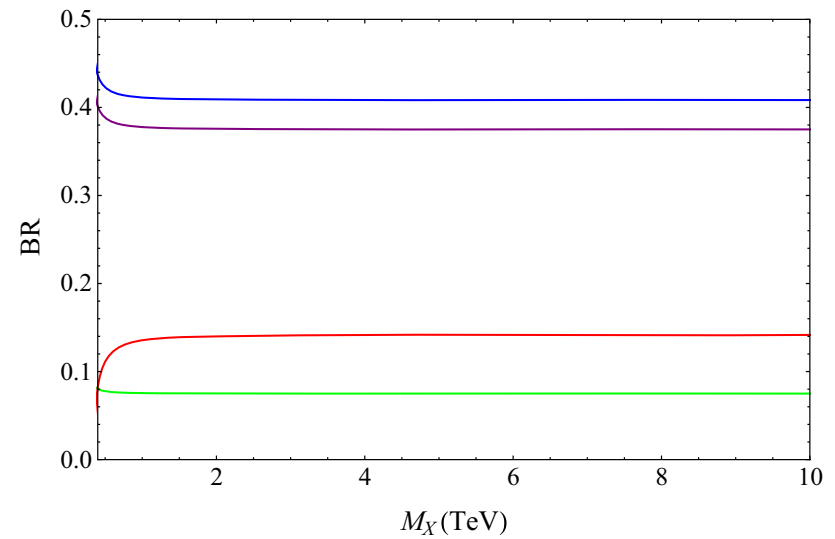

Fig. 2 Branching ratios of the gauge boson $X_{\mu}$ as the functions of its mass $M_{X}$. The green, purple, blue, and red curves correspond to $X \rightarrow \bar{v} v, \bar{l} l, \bar{q} q, \bar{t} t$, respectively

This shows that the decay channels $X \rightarrow l^{+} l^{-}$and $X \rightarrow \bar{q} q$ ( $q=u, d, s, c, b)$ are the dominant ones and thus the decays of the gauge boson $X$ can be accessible via these channels.

The gauge boson $X$ should contribute to the cross sections of $e^{+} e^{-} \rightarrow f^{+} f^{-}$performed at LEP-II. For $M_{X}$ much larger than the collider energy $\sqrt{s}$, it should lead to the effective four-fermion interactions induced by the exchange of the gauge boson $X$ as

$$
\begin{aligned}
\mathcal{L}_{\text {eff }}= & \frac{1}{1+\delta_{e f}} \frac{1}{M_{X}^{2}}\left[\bar{e} \gamma^{\mu}\left(C_{e, V}+\gamma_{5} C_{e, A}\right) e\right] \\
& \times\left[\bar{f} \gamma_{\mu}\left(C_{f, V}+\gamma_{5} C_{f, A}\right) f\right],
\end{aligned}
$$

where $\delta_{e f}=1(0)$ for $f=e(f \neq e)$, the vector and axial coupling factors are determined as

$C_{f, V}=\frac{\lambda_{f_{L}}+\lambda_{f_{R}}}{2}, \quad C_{f, A}=\frac{\lambda_{f_{R}}-\lambda_{f_{L}}}{2}$.

The LEP-II data of $e^{+} e^{-} \rightarrow e^{+} e^{-}, \mu^{+} \mu^{-}, \tau^{+}, \tau^{-}$, in Ref. [79], puts the constraint on the gauge boson $X$ as

$\frac{2 \sqrt{\pi} M_{X}}{\sqrt{C_{f, V}^{2}+C_{f, A}^{2}}} \gtrsim 24.6 \mathrm{TeV}$,

where $C_{f, V}=-\frac{3 x}{2} g_{X}$ and $C_{f, A}=\frac{x}{2} g_{X}$ for $f=e, \mu, \tau$. This leads to a bound on the $\mathrm{U}(1)_{X}$ breaking scale

$\frac{M_{X}}{g_{X}}=v^{\prime} \gtrsim|x| \times 10.97 \mathrm{TeV}$.

Using the relation (32), we obtain the bound for the ratio of the mass $M_{X}$ and the scale $\Lambda$ as

$\frac{M_{X}}{\Lambda} \gtrsim \frac{2.26}{T_{0}}|x| \times 10^{-15}$.

On the contrary, if $M_{X}<\sqrt{s}$, the gauge boson $X$ should appear as a $\bar{f} f$ resonance and the corresponding coupling is constrained by LEP-II data $[69,79]$ as

$\sqrt{C_{f, V}^{2}+C_{f, A}^{2}} \lesssim 10^{-2} \longrightarrow g_{X} \lesssim \frac{1}{|x|} \sqrt{\frac{2}{5}} \times 10^{-2}$.

This leads to a bound on the scale $\Lambda / T_{0}$ as

$\frac{\Lambda}{T_{0}} \lesssim \frac{3.07}{|x|} \times 10^{16} \mathrm{GeV}$.

This bound reveals the constraint on the bulk radius as

$R \gtrsim 0.65|x| \times 10^{-30} \mathrm{~cm}$.

In particular, the atomic parity violation in Cesium $\left({ }_{55}^{133} \mathrm{Cs}\right)$ imposes very strong constraints on the couplings of the gauge boson $X$ to the electron and the quarks $u$ and $d$. The measurement of the nuclear weak charge of Cesium is given by [80-85]

$Q_{W}^{\exp }\left({ }_{55}^{133} \mathrm{Cs}\right)=-73.16(29)_{\exp }(20)_{\mathrm{th}}$,

where the first and second uncertainties are experimental and theoretical, respectively. This measurement is perfect agreement with the prediction of the SM (including electroweak radiative corrections) as [86-90]

$Q_{W}^{\text {th }}\left({ }_{55}^{133} \mathrm{Cs}\right)=-73.16(3)$.

It thus requires the contributions of the new physics to the nuclear weak charge of Cesium satisfying, $\left|\Delta Q_{W}\left({ }_{55}^{133} \mathrm{Cs}\right)\right| \lesssim$ 0.52 . The correction to the nuclear weak charge of given isotope, which is mediated by a massive gauge boson, is computed in Ref. [91]. Using this result, $\Delta Q_{W}\left({ }_{55}^{133} \mathrm{Cs}\right)$ is given in this model as

$$
\begin{aligned}
\Delta Q_{W}\left({ }_{55}^{133} \mathrm{Cs}\right)=- & 16\left(\frac{M_{Z}}{M_{X}}\right)^{2}\left(\frac{\cos \theta_{W}}{g}\right)^{2} C_{e, A} \\
& \times\left[(2 Z+N) C_{u, V}+(Z+2 N) C_{d, V}\right],
\end{aligned}
$$

where $M_{Z}$ is the mass of the gauge boson $Z, \theta_{W}$ is the Weinberg angle, $Z=55$ and $N=78$. Then, we obtain a bound as

$\frac{M_{X}}{g_{X}}=v^{\prime} \gtrsim|x| \times 7.3 \mathrm{TeV}$,

which is a stronger constraint compared to the LEP-II data. The corresponding bound on the ratio of the mass $M_{X}$ and the scale $\Lambda$ is given as

$\frac{M_{X}}{\Lambda} \gtrsim \frac{1.5}{T_{0}}|x| \times 10^{-15}$.

Now we arrive at analyzing the contribution of the gauge boson $X$ to the anomalous magnetic moment of the muon. The deviation between the experiment and theory is now $[92,93]$

$$
\Delta a_{\mu}=a_{\mu}^{\exp }-a_{\mu}^{\text {th }}=306 \pm 72 \times 10^{-11},
$$



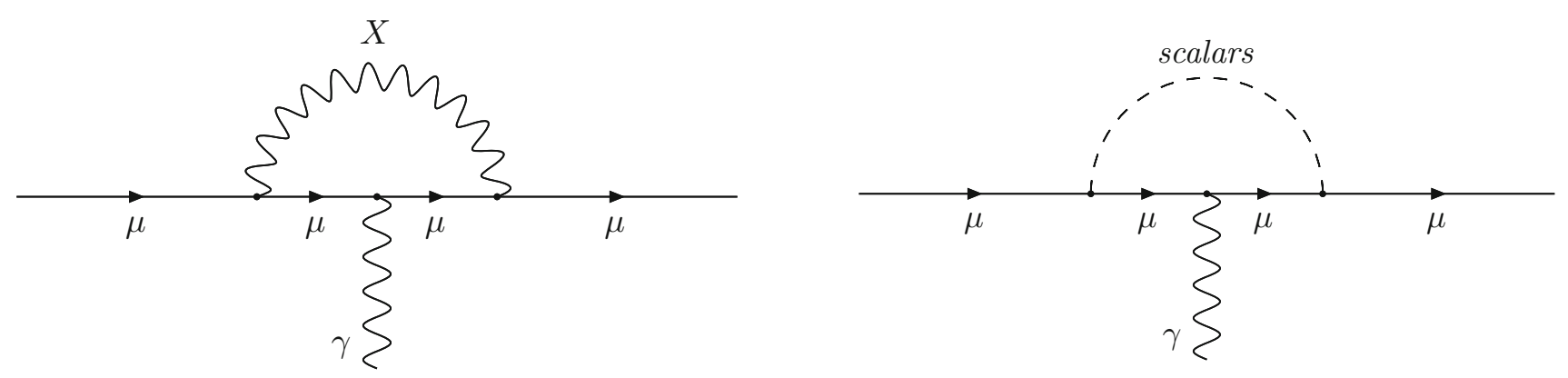

Fig. 3 Feynman diagrams for the muon anomalous magnetic moment with the new contributions of the gauge boson $X$ and the scalars

which is at $4.3 \sigma$. In this model, the new contributions to the anomalous magnetic moment of the muon are generated by one-loop diagrams mediated by the gauge boson $X$, the exotic Higgs $h_{2}$, the neutral KK excitations $\left\{\phi_{1 n}^{0}, \phi_{2 n}^{0}\right\}$, as shown in Fig. 3. (Note that, $\left\{\phi_{1 n}^{0}, \phi_{2 n}^{0}\right\}$ are neutral components of the KK doublets $\left\{\phi_{1 n}, \phi_{2 n}\right\}$, respectively.)

However, the contribution of the scalars is proportional to $h_{\mu}^{2} \approx 10^{-8}$ where $h_{\mu}$ is the Yukawa coupling constant of the muon, thus their contribution is small and can be neglected compared to that of the gauge boson $X$. The contribution of the gauge boson $X$ reads $[94,95]$

$$
\begin{aligned}
\Delta a_{\mu}^{X}= & \frac{1}{4 \pi^{2}}\left(\frac{m_{\mu}}{M_{X}}\right)^{2} \\
& \times\left[C_{\mu, V}^{2} L_{V}\left(\frac{m_{\mu}}{M_{X}}\right)+C_{\mu, A}^{2} L_{A}\left(\frac{m_{\mu}}{M_{X}}\right)\right],
\end{aligned}
$$

where $m_{\mu}$ is the muon mass, and the functions $L_{V, A}(\lambda)$ are defined as

$$
\begin{aligned}
& L_{V}(\lambda)=\int_{0}^{1} d z \frac{z^{2}(1-z)}{1-z+\lambda^{2} z^{2}}, \\
& L_{A}(\lambda)=\int_{0}^{1} d z \frac{\left(z-z^{2}\right)(z-4)-2 \lambda^{2} z^{3}}{1-z+\lambda^{2} z^{2}} .
\end{aligned}
$$

For $M_{X} \gg m_{\mu}$ or $\lambda \ll 1$, we have the approximation, $L_{V}(\lambda) \simeq L_{V}(0)=0.333$ and $L_{A}(\lambda) \simeq L_{A}(0)=-0.283$. With the bound (95), the contribution of the gauge boson $X$ to the anomalous magnetic moment of the muon is constrained as

$\Delta a_{\mu}^{X} \lesssim 14.3 \times 10^{-11}$.

Compared to (97), clearly the contribution of the gauge boson $X$ is not enough responsible for the deviation between the experiment and the theory. On the other hand, this deviation should be explained by new physics beyond this model.

\subsection{Discrimination and implications}

If the signal of a new neutral gauge boson $Z^{\prime}$ associated with $\mathrm{U}(1)^{\prime}$ is discovered at the accelerators, the next task will be to specify the underlying model which predicts it.
Table 2 The $Z^{\prime}$ couplings to the SM fermions for the models generated from $\mathrm{E}_{6}$ and $\mathrm{SO}(10)$, with an overall $e / \cos \theta_{W}$ factored out and $\alpha_{L R} \simeq$ 1.59

\begin{tabular}{lcccc}
\hline & $\chi$ & $\psi$ & $\eta$ & LR \\
\hline$q_{L}$ & $-\frac{1}{2 \sqrt{6}}$ & $\frac{\sqrt{10}}{12}$ & $\frac{1}{3}$ & $-\frac{1}{6 \alpha_{L R}}$ \\
$u_{R}$ & $\frac{1}{2 \sqrt{6}}$ & $-\frac{\sqrt{10}}{12}$ & $-\frac{1}{3}$ & $-\frac{1}{6 \alpha_{L R}}+\frac{\alpha_{L R}}{2}$ \\
$d_{R}$ & $-\frac{3}{2 \sqrt{6}}$ & $-\frac{\sqrt{10}}{12}$ & $\frac{1}{6}$ & $-\frac{1}{6 \alpha_{L R}}-\frac{\alpha_{L R}}{2}$ \\
$l_{L}$ & $\frac{3}{2 \sqrt{6}}$ & $\frac{\sqrt{10}}{12}$ & $-\frac{1}{6}$ & $\frac{1}{2 \alpha_{L R}}$ \\
$e_{R}$ & $\frac{1}{2 \sqrt{6}}$ & $-\frac{\sqrt{10}}{12}$ & $-\frac{1}{3}$ & $-\frac{1}{2 \alpha_{L R}}-\frac{\alpha_{L R}}{2}$ \\
\hline
\end{tabular}

Many studies of the gauge boson $Z^{\prime}$ focus the $\mathrm{U}(1)^{\prime}$ models which occur in the decomposition of GUTs, based on the exceptional group $\mathrm{E}_{6}$ and the group $\mathrm{SO}(10)$ [23-28]. Since, here we are interested in distinguishing our model to these models.

The $\mathrm{U}(1)^{\prime}$ models originating from the exceptional group $\mathrm{E}_{6}$ are obtained by the following breaking chain

$$
\begin{aligned}
\mathrm{E}_{6} & \rightarrow \mathrm{SO}(10) \times \mathrm{U}(1)_{\psi} \\
& \rightarrow \mathrm{SU}(5) \times \mathrm{U}(1)_{\chi} \times \mathrm{U}(1)_{\psi} \rightarrow \mathrm{SU}(5) \times \mathrm{U}(1)_{\beta},
\end{aligned}
$$

where the boson $Z^{\prime}$ is identified as

$Z^{\prime}=Z_{\chi} \cos \beta+Z_{\psi} \sin \beta$,

with $\beta$ to be a free parameter in the breaking scheme. From this, we obtain the $\psi$ model, the $\chi$ model, and the $\eta$ model which correspond to $\beta=0, \beta=\pi / 2$, and $\beta=\arctan (-\sqrt{5 / 3})$ (the special case often considered), respectively. The breaking of the group $\mathrm{SO}(10)$ leads to the left-right (LR) symmetric model as

$\mathrm{SO}(10) \rightarrow \mathrm{SU}(3)_{C} \times \mathrm{SU}(2)_{L} \times \mathrm{SU}(2)_{R} \times \mathrm{U}(1)_{B-L} \cdot(10$

The $Z^{\prime}$ couplings to the SM fermions in these models are summarized in Table 2 [96].

In principle, the models predicting $Z^{\prime}$ are distinguished by the $Z^{\prime}$ couplings to the fermions, $Z^{\prime}$ mass or $\mathrm{U}(1)^{\prime}$ breaking scale, the total decay width $\Gamma_{Z^{\prime}}$, the branching ratios of the decay $Z^{\prime}$, the cross section to the pair $e^{+} e^{-}$, as well as 
Table 3 The ratio between BR of the top quark and BR of all quarks

\begin{tabular}{cccccc}
\hline & $\chi$ & $\psi$ & $\eta$ & $\mathrm{LR}$ & $U(1)_{X}$ \\
\hline$\frac{\mathrm{BR}}{\mathrm{BR}_{\text {total }}}$ & $\simeq 5.55 \%$ & $\simeq 16.66 \%$ & $\simeq 20.51 \%$ & $\simeq 12.42 \%$ & $\simeq 25.82 \%$ \\
\hline
\end{tabular}

the forward-backward asymmetry. In particular, the discovered $Z^{\prime}$ couplings to the SM fermions can be studied with a reasonable accuracy, thus they provide the potential way to determine the underlying model from which $Z^{\prime}$ arises. The studies of the $Z^{\prime}$ coupling extractions are performed in many works $[67,68,96,97]$. In GUTs as mentioned above, the coupling constants of the SM and the extra U(1)'s are determined by the coupling constant of GUTs. In this way, it can express the coupling constant of the $Z^{\prime}$ couplings to the $\mathrm{SM}$ fermions in terms of the coupling constant of the electromagnetic interaction and the Weinberg angle. As a result, in the $\mathrm{U}(1)^{\prime}$ models generated from GUTs, the coupling constant of the $Z^{\prime}$ couplings to the SM fermions is completely fixed by the values of the the electromagnetic coupling constant and the Weinberg angle. In our framework, the coupling constant of the $Z^{\prime}$ couplings to the SM fermions is expressed in terms of the inversion of the bulk radius, $R^{-1}=\Lambda / T_{0}$, as seen in (32). And, since it is completely fixed by the difference between the mass of the zero mode and that of its first $\mathrm{KK}$ excitation. Another interesting property of the boson $Z^{\prime}$, which can be used to determine the underlying model from which $Z^{\prime}$ arises, is the ratio between $\operatorname{BR}(X \rightarrow \bar{t} t)$ of the top quark and $\sum_{q=u, d, s, c, b, t} \operatorname{BR}(X \rightarrow \bar{q} q)$ of all quarks, for the mass of $Z^{\prime}$ much larger than $m_{t}$. We present the value of this ratio corresponding to our model and the $U(1)^{\prime}$ models occurring in the $\mathrm{E}_{6}$-type and $\mathrm{SO}(10)$-type GUTs in Table 3.

A question which we can ask is whether this framework features unification schemes which interpret nongravitational interactions as a result of the non-trivial structure of the spacetime. In order to answer this question, now let us consider another scenario at which the gauge field $X_{\mu}$ is identified as the gauge field associated with the hypercharge gauge group $\mathrm{U}(1)_{Y}$ of the SM. On the other hand, the emergent gauge group $\mathrm{U}(1)_{X}$, investigated above, is identified as the gauge group $\mathrm{U}(1)_{Y}$. Since the gauge symmetry group of theory is only $\mathrm{SU}(3)_{C} \otimes \mathrm{SU}(2)_{L}$. For simplicity, the fermion content is given as in (41) but not to have the right-handed neutrinos $N_{a R}$. Their $\theta$-dependence is given in (44), but there the quantum number $X$ is identified as hypercharge. With respect to the scalar sector, the scalar field given in (60) is identified as the doublet of the symmetry group $\mathrm{SU}(2)_{L}$. Similarly, one can determine the relation between the gauge coupling constant $g^{\prime}$ associated with $\mathrm{U}(1)_{Y}$ and the bulk radius $R$ as, $g^{\prime}=\frac{\sqrt{2}}{M_{P l} R}$. This implies that $R^{-1}$ is close to the Planck energy scale or in other words the size of the fibers must be extremely small. Furthermore, we can deter- mine the hypercharge of the fermions from the absence of the nontrivial anomalies $\left\{\left[\mathrm{SU}(3)_{C}\right]^{2} \mathrm{U}(1)_{X},\left[\mathrm{SU}(2)_{L}\right]^{2} \mathrm{U}(1)_{X}\right.$, $\left.\left[\mathrm{U}(1)_{X}\right]^{3},[\text { Gravity }]^{2} \mathrm{U}(1)_{X}\right\}$. For the lepton doublet $l_{a}$, the right-handed electron $e_{a R}$, and the quark doublet $q_{a}$, their hypercharge is obtained as

$X_{l_{a}}=-x, \quad X_{e_{a R}}=-2 x, \quad X_{q_{a}}=\frac{x}{3}$.

Whereas, for the right-handed up quark $u_{a R}$ and right-handed down quark $d_{a R}$, their hypercharge belongs one of two solution sets as

$X_{u_{a R}}=\frac{4 x}{3}, \quad X_{d_{a R}}=-\frac{2 x}{3}$,

or

$X_{u_{a R}}=-\frac{2 x}{3}, \quad X_{d_{a R}}=\frac{4 x}{3}$.

The first solution set (105) and $x=1 / 2$ are selected by imposing that the left-handed and right-handed particles of the same type must transform the same way under a combination of the weak isospin $T_{3}$ and the hypercharge $X$. Thus, this framework is possible to interpret the interaction associated with the hypercharge gauge group $\mathrm{U}(1)_{Y}$ as a result of the non-trivial structure of the spacetime. For generalizing this unification for both $\mathrm{SU}(3)_{C}$ and $\mathrm{SU}(2)_{L}$, the fiber should be a Lie group manifold $\mathrm{SU}(3) \otimes \mathrm{SU}(2) \otimes \mathrm{U}(1)$. However, in order to claim whether this framework provides the unification for both $\mathrm{SU}(3)_{C}$ and $\mathrm{SU}(2)_{L}$, we need to treat more care and detail which is beyond the scope of the present work.

Let us see whether there has any good relic candidate in this model. In this model, apart from the gauge boson $X$, there are many new particles as follows: the sterile neutrinos $v_{a R}^{\prime}$ and their KK partners $\left\{\psi_{n a R}^{\prime}, \chi_{n a R}^{\prime}\right\}$, the exotic Higgs $h_{2}$, and the KK excitations $\left\{\phi_{1 n}, \phi_{2 n}\right\}$ which originate from the bulk scalar doublet $H$. Obviously, the exotic Higgs $h_{2}$ is unstable, because it is more (much) heavy than the SM Higgs $h_{1}$ and thus it should decay into $h_{1}$ through the coupling, $\simeq$ $\lambda_{3} v^{\prime} c_{\alpha}^{3} h_{1}^{2} h_{2}$. In order to see that the remaining new particles are also unstable, we write the couplings, obtained from (72), as

$$
\begin{aligned}
\mathcal{L}_{1} \simeq & {\left[\frac{h_{a b}^{v} c_{\alpha}}{\sqrt{2}}{\overline{v^{\prime}}}_{a L} h_{1} v_{b R}^{\prime}+\frac{h_{n a b}^{\psi} c_{\alpha}}{\sqrt{2}}{\overline{v^{\prime}}}_{a L} h_{1} \psi_{n b R}^{\prime}\right.} \\
& \left.+\frac{h_{n a b}^{\chi} c_{\alpha}}{\sqrt{2}}{\overline{v^{\prime}}}_{a L} h_{1} \chi_{n b R}^{\prime}+\text { H.c. }\right], \\
\mathcal{L}_{2}= & h_{n a b}^{e} \bar{b}_{a} e_{b R} \phi_{1 n}+h_{n a b}^{d} \bar{q}_{a} d_{b R} \phi_{1 n} \\
& +h_{n a b}^{u} \bar{q}_{a} u_{b R} \widetilde{\phi}_{1 n}-i \frac{h_{n a b}^{e}{ }^{e}}{x} \bar{l}_{a} e_{b R} \phi_{2 n} \\
& -i \frac{h_{n a b}^{d} n}{x} \bar{q}_{a} d_{b R} \phi_{2 n}+i \frac{h_{n a b}^{u} n}{x} \bar{q}_{a} u_{b R} \widetilde{\phi}_{2 n}+\text { H.c., }
\end{aligned}
$$




$$
\begin{aligned}
\mathcal{L}_{3}=h_{n a b}^{v} \bar{l}_{a} v_{b R}^{\prime} \widetilde{\phi}_{1 n}+ & \frac{i h_{n a b}^{v}}{x} \bar{l}_{a} v_{b R}^{\prime} \widetilde{\phi}_{2 n} \\
& +h_{m n a b}^{(1)} \bar{l}_{a} \psi_{b m}^{\prime} \widetilde{\phi}_{1 n}+h_{m n a b}^{(2)} \bar{l}_{a} \chi_{b m}^{\prime} \widetilde{\phi}_{1 n} \\
& +h_{m n a b}^{(3)} \bar{l}_{a} \psi_{b m}^{\prime} \widetilde{\phi}_{2 n}+h_{m n a b}^{(4)} \bar{l}_{a} \chi_{b m}^{\prime} \widetilde{\phi}_{2 n}+\text { H.c. },
\end{aligned}
$$

where $\widetilde{\phi}_{1 n}=i \sigma^{2} \phi_{1 n}^{*}, \widetilde{\phi}_{2 n}=i \sigma^{2} \phi_{2 n}^{*}$, and

$$
\begin{aligned}
& h_{n a b}^{e}=\frac{i x\left(1-e^{-2 \pi i x}\right) \bar{h}_{a b}^{e}}{2 \pi \sqrt{\pi R}\left(n^{2}-x^{2}\right)}, \\
& h_{n a b}^{d}=\frac{i x\left(1-e^{-2 \pi i x}\right) \bar{h}_{a b}^{d}}{2 \pi \sqrt{\pi R}\left(n^{2}-x^{2}\right)}, \\
& h_{n a b}^{u}=-\frac{i x\left(1-e^{2 \pi i x}\right) \bar{h}_{a b}^{u}}{2 \pi \sqrt{\pi R}\left(n^{2}-x^{2}\right)}, \\
& h_{n a b}^{v}=-\frac{i x\left(1-e^{2 \pi i x}\right) \bar{h}_{a b}^{v}}{2 \pi \sqrt{\pi R}\left(n^{2}-x^{2}\right)}, \\
& h_{m n a b}^{(1)}=-\frac{i x\left(1-e^{2 \pi i x}\right) \bar{h}_{a b}^{v}}{\pi \sqrt{2 \pi R}} \\
& \times \frac{m^{2}+n^{2}-x^{2}}{\left[(m+n)^{2}-x^{2}\right]\left[(m-n)^{2}-x^{2}\right]}, \\
& h_{m n a b}^{(2)}=\frac{\left(1-e^{2 \pi i x}\right) \bar{h}_{a b}^{v}}{\pi \sqrt{2 \pi R}} \\
& \times \frac{m\left(m^{2}-n^{2}-x^{2}\right)}{\left[(m+n)^{2}-x^{2}\right]\left[(m-n)^{2}-x^{2}\right]}, \\
& h_{m n a b}^{(3)}=\frac{\left(1-e^{2 \pi i x}\right) \bar{h}_{a b}^{v}}{\pi \sqrt{2 \pi R}} \\
& \times \frac{n\left(n^{2}-m^{2}-x^{2}\right)}{\left[(m+n)^{2}-x^{2}\right]\left[(m-n)^{2}-x^{2}\right]}, \\
& h_{m n a b}^{(4)}=-\frac{\left(1-e^{2 \pi i x}\right) \bar{h}_{a b}^{v}}{\pi \sqrt{2 \pi R}} \\
& \times \frac{m n x}{\left[(m+n)^{2}-x^{2}\right]\left[(m-n)^{2}-x^{2}\right]} .
\end{aligned}
$$

Note that, because the free parameter $x$ satisfies the condition (75), all the coupling constant in (108) are always different to zero. $\mathcal{L}_{1}$ describes the couplings of the sterile neutrinos $v_{a R}^{\prime}$ and their KK partners $\left\{\psi_{n a R}^{\prime}, \chi_{n a R}^{\prime}\right\}$ to the SM leptons and the SM Higgs $h_{1} \cdot \mathcal{L}_{2}$ describes the couplings of the KK excitations $\left\{\phi_{1 n}, \phi_{2 n}\right\}$ to the SM fermions. Finally, $\mathcal{L}_{3}$ describes the couplings which $\left\{v_{a R}^{\prime}, \psi_{n a R}^{\prime}, \chi_{n a R}^{\prime}, \phi_{1 n}, \phi_{2 n}\right\}$ couple to with together and to the SM leptons. Because of the presence of these couplings, the sterile neutrinos $v_{a R}^{\prime}$ and all KK excitations will decay into the SM particles. In this sense, there has no any good relic candidate in this model. However, this model may contain a good relic candidate and thus may account for dark matter, if the scalar sector of this model is extended at which we introduce additionally an inert scalar field $\Phi^{\prime}$ similar to $\Phi$ but with $X_{\Phi^{\prime}} \neq X_{\Phi}$. Because both $\Phi$ and $\Phi^{\prime}$ have no the Yukawa couplings to the fermions, the action of theory is accidentally invariant under the $Z_{2}$ transformation

$\Phi \longrightarrow-\Phi, \quad \Phi^{\prime} \longrightarrow-\Phi^{\prime}$

All the other fields are even under the $Z_{2}$ transformation. We assume that $\left\langle\Phi^{\prime}\right\rangle=0$ (and thus $\Phi^{\prime}$ is realized as the inert scalar) whereas other scalars all develop non-vanishing VEVs. Here, some conditions must be required to guarantee that the inert vacuum is the global minimum of the scalar potential. As a result, after the breaking symmetry, by the exactly conserved $Z_{2}$ symmetry only $\Phi^{\prime}$ is always coupled in pairs in the interactions. Since $\Phi^{\prime}$ is stable and may be responsible for dark matter. Note that, $\Phi^{\prime}$ couples to the SM particles via mostly the gauge boson $X$ and the Higgs $h_{1}$. A detail study of dark matter in this model will be taken in our upcoming work.

\section{Conclusion}

In this paper, we have proposed a $\mathrm{U}(1)_{X}$ extension of the SM, which departs fundamentally from the theory in the fivedimensional fiber bundle spacetime with the SM gauge symmetry group. Here, the base manifold and fiber of the spacetime are the four-dimensional Minkowski-flat manifold and the Lie group manifold U(1), respectively. The local gauge transformation under $\mathrm{U}(1)_{X}$ is originated from the general transformation in the fiber coordinate of the spacetime. The $\mathrm{U}(1)_{X}$ charges of the SM fermions are the quantum number characterizing the active action of the Lie group U(1) on the corresponding fundamental fermions. In particular, this scenario allows to explain naturally the small masses of the neutrinos as a result of the short-distance structure of the spacetime.

We have studied the tree-level decays of the new gauge boson into the two-body final states, at which its branching ratios and dominant decay channels are discussed. The bound on the $\mathrm{U}(1)_{Y}$ breaking scale or the $\mathrm{U}(1)_{X}$ coupling constant has been obtained from the LEP-II constraints. In particular, the atomic parity violation in Cesium $\left({ }_{55}^{133} \mathrm{Cs}\right)$ imposes a very strong constraint on the $\mathrm{U}(1)_{X}$ breaking scale. Finally, we have analyzed the contribution of the new gauge boson to the anomalous magnetic moment of the muon, which requires additional contributions from new physics beyond this model.

Acknowledgements We would like to express sincere gratitude to the referee for his constructive comments and suggestions which have helped us to improve the quality of the paper. 
Data Availability Statement This manuscript has no associated data or the data will not be deposited. [Authors' comment: This is a theoretical work, thus there has no data associated with this article.]

Open Access This article is distributed under the terms of the Creative Commons Attribution 4.0 International License (http://creativecomm ons.org/licenses/by/4.0/), which permits unrestricted use, distribution, and reproduction in any medium, provided you give appropriate credit to the original author(s) and the source, provide a link to the Creative Commons license, and indicate if changes were made.

Funded by SCOAP ${ }^{3}$.

\section{Appendix A: Deriving a covariant basic of the horizontal tangent subspace}

In this appendix, we would like to obtain a covariant basic of the horizontal tangent subspace. The horizontal tangent subspace $H_{p} M_{5}$, at any point $p \in M_{5}$, is determined as the kernel of a Lie-algebra-valued connection one-form $\omega$ [78], meaning that

$H_{p} M_{5}=\left\{V \in T_{p} M_{5} \mid \omega(V)=0\right\}$,

where $T_{p} M_{5}$ is the tangent space of $M_{5}$ at the point $p$. The local expression of $\omega$ is defined on $M_{5}$ by [78]

$$
\begin{aligned}
\omega & =g^{-1} X(x) g+g^{-1} d g, \\
& =i\left[d \theta+g_{X} X_{\mu}(x) d x^{\mu}\right],
\end{aligned}
$$

where $g=e^{i \theta}$ is the fiber coordinate, $X(x)=i g_{X} X_{\mu}(x) d x^{\mu}$, and $d$ is the exterior differential operator. Note that, under the general coordinate transformation (2) because $\omega^{\prime}\left(x^{\prime}, e^{i \theta^{\prime}}\right)=$ $\omega\left(x, e^{i \theta}\right)$, the field $X_{\mu}$ should transform in the rule (16). For $V$ to be a horizontal tangent vector, determined by an integral curve $\left(x^{\mu}(t), e^{i \theta(t)}\right)$, we have

$V=\frac{d x^{\mu}(t)}{d t} \frac{\partial}{\partial x^{\mu}}+\frac{d \theta(t)}{d t} \frac{\partial}{\partial \theta}$.

Also, we have $\omega(V)=0$, leading to

$\frac{d \theta(t)}{d t}+g_{X} X_{\mu}(x) \frac{d x^{\mu}(t)}{d t}=0$.

From Eqs. (A3) and (A4), we find

$V=\frac{d x^{\mu}(t)}{d t}\left(\frac{\partial}{\partial x^{\mu}}-g_{X} X_{\mu} \frac{\partial}{\partial \theta}\right)$.

This clearly implies

$\left\{\frac{\partial}{\partial x^{\mu}}-g_{X} X_{\mu} \frac{\partial}{\partial \theta} \equiv \hat{\partial}_{\mu}\right\}$,

to be a basic of the horizontal tangent subspace. It is easily to see that the basic $\left\{\hat{\partial}_{\mu}\right\}$ transforms under the general coordinate transformation (2) as follows:

$\hat{\partial}_{\mu} \longrightarrow \hat{\partial}_{\mu}^{\prime}=\frac{\partial x^{\nu}}{\partial x^{\prime \mu}} \hat{\partial}_{\nu}$ which is covariant. Thus, $\left\{\hat{\partial}_{\mu}\right\}$ is actually a covariant basic of the horizontal tangent subspace.

\section{Appendix B: The anomaly cancellation}

In Sect. 3, we have built a realistic model which consists of a set of fields, propagating dynamically in the fivedimensional fiber bundle spacetime, respecting for the gauge symmetry group $\mathrm{SU}(3)_{C} \otimes \mathrm{SU}(2)_{L} \otimes \mathrm{U}(1)_{Y}$. From this, we have obtained an extension of the SM, in effective fourdimensional spacetime, with the gauge symmetry group $\mathrm{SU}(3)_{C} \otimes \mathrm{SU}(2)_{L} \otimes \mathrm{U}(1)_{Y} \otimes \mathrm{U}(1)_{X}$. Here, the SM fermions charge under the gauge symmetry group $\mathrm{U}(1)_{X}$ as

$$
\begin{aligned}
& X_{v_{a L}}=X_{e_{a L}}=X_{L}, \\
& X_{e_{a R}}=X_{E}, \\
& X_{u_{a L}}=X_{d_{a L}}=X_{Q}, \\
& X_{u_{a R}}=X_{U}, \\
& X_{d_{a R}}=X_{D},
\end{aligned}
$$

where the quantum number $\left\{X_{L}, X_{E}, X_{Q}, X_{U}, X_{D}\right\}$ are constrained by the anomaly cancellation. Also, there are new fermions as the right-handed neutrinos $v_{a R}$ and KK excitations $\left\{\psi_{n a R}, \chi_{n a R}\right\}$. We can list six nontrivial anomalies associated with $\mathrm{U}(1)_{X}$ as follows: $\left[\mathrm{SU}(3)_{C}\right]^{2} \mathrm{U}(1)_{X}$, $\left[\mathrm{SU}(2)_{L}\right]^{2} \mathrm{U}(1)_{X}, \quad\left[\mathrm{U}(1)_{Y}\right]^{2} \mathrm{U}(1)_{X}, \quad \mathrm{U}(1)_{Y}\left[\mathrm{U}(1)_{X}\right]^{2}$, $\left[\mathrm{U}(1)_{X}\right]^{3}$, and $[\text { Gravity }]^{2} \mathrm{U}(1)_{X}$. Note that, the anomalies associated with the SM gauge symmetry group vanish all. First, we consider the vanishing anomaly $\left[\mathrm{SU}(3)_{C}\right]^{2} \mathrm{U}(1)_{X}$ which is given as

$$
\left[\mathrm{SU}(3)_{C}\right]^{2} \mathrm{U}(1)_{X} \sim 2 \times 3 X_{Q}-3 X_{U}-3 X_{D}=0 .
$$

The vanishing second anomaly $\left[\mathrm{SU}(2)_{L}\right]^{2} \mathrm{U}(1)_{X}$ is written as

$$
\left[\mathrm{SU}(2)_{L}\right]^{2} \mathrm{U}(1)_{X} \sim 2 \times 3 X_{Q}+2 X_{L}=0
$$

The vanishing third anomaly $\left[\mathrm{U}(1)_{Y}\right]^{2} \mathrm{U}(1)_{X}$ leads to

$$
\begin{aligned}
& {\left[\mathrm{U}(1)_{Y}\right]^{2} \mathrm{U}(1)_{X} \sim 2 \times 3 \times\left(\frac{1}{6}\right)^{2} X_{Q}} \\
& +2 \times\left(-\frac{1}{2}\right)^{2} X_{L}-3 \times\left(\frac{2}{3}\right)^{2} X_{U} \\
& -3 \times\left(-\frac{1}{3}\right)^{2} X_{D}-(-1)^{2} X_{E}=0 .
\end{aligned}
$$

With the vanishing fourth anomaly $\mathrm{U}(1)_{Y}\left[\mathrm{U}(1)_{X}\right]^{2}$, we have

$$
\begin{gathered}
\mathrm{U}(1)_{Y}\left[\mathrm{U}(1)_{X}\right]^{2} \sim 2 \times 3 \times \frac{1}{6} X_{Q}^{2}+2 \times\left(-\frac{1}{2}\right) X_{L}^{2} \\
-3 \times \frac{2}{3} X_{U}^{2}-3 \times\left(-\frac{1}{3}\right) X_{D}^{2}-(-1) X_{E}^{2}=0 .
\end{gathered}
$$


Note that, with respect to the first four anomalies, because the right-handed neutrinos $v_{a R}$ and their KK counter parts $\left\{\psi_{n a R}, \chi_{n a R}\right\}$ do not charge under the SM gauge symmetry group, thus they do not contribute to these anomalies. The vanishing fifth anomaly $\left[\mathrm{U}(1)_{X}\right]^{3}$ is given by

$$
\left[\mathrm{U}(1)_{X}\right]^{3} \sim 2 \times 3 X_{Q}^{3}+2 X_{L}^{3}-3 X_{U}^{3}-3 X_{D}^{3}-X_{E}^{3}=0 .
$$

The vanishing last anomaly [Gravity $]^{2} \mathrm{U}(1)_{X}$ leads to

$$
\begin{aligned}
& {[\text { Gravity }]^{2} \mathrm{U}(1)_{X} \sim 2 \times 3 X_{Q}+2 X_{L}-3 X_{U}} \\
& -3 X_{D}-X_{E}=0 .
\end{aligned}
$$

For last two anomalies, the right-handed neutrinos $v_{a R}$ do not charge under the gauge symmetry group $\mathrm{U}(1)_{X}$, thus they do not contribute to these anomalies. Whereas, for the KK excitations $\left\{\psi_{n a R}, \chi_{n a R}\right\}$, they couple to the gauge boson $X_{\mu}$ in a unusual way given at (57). Thus, these KK excitations should not appear in the triangle diagrams which have three $X_{\mu}$ gauge bosons or one $X_{\mu}$ gauge boson. By solving Eqs. (B2)-(B7), one find

$$
\begin{gathered}
X_{L}=-x, \quad X_{E}=-2 x, \quad X_{Q}=\frac{1}{3} x, \\
X_{U}=\frac{4}{3} x, \quad X_{D}=-\frac{2}{3} x,
\end{gathered}
$$

where $x$ is a free parameter.

\section{References}

1. S.W. Hawking, G.F.R. Ellis, The large scale structure of spacetime (Cambridge University Press, Cambridge, 1973)

2. T. Kaluza, Sitzungsber. Preuss. Akad. Wiss. Berlin (Math. Phys.) 1921: 966972 (1921)

3. O. Klein, Zeitschrift fr Physik a Hadrons and Nuclei 37(12), 895906 (1926). https://doi.org/10.1007/BF01397481

4. B. de Witt, Dynamical Theories of Groups and Fields (Gordon and Breach, New York, 1965)

5. R. Kerner, Annales de l'Institut Henri Poincar 9, 143 (1968)

6. A. Trautman, Rep. Math. Phys. 1, 29 (1970)

7. Y.M. Cho, J. Math. Phys. 16, 2029 (1975)

8. Y.M. Cho, P.G.O. Freund, Phys. Rev. D 12, 1771 (1975)

9. E. Witten, Nucl. Phys. B 186, 412 (1981)

10. D. Bailin, A. Love, Rep. Prog. Phys. 50, 1087 (1987)

11. J.M. Overduin, P.S. Wesson, Phys. Rep. 283, 303 (1997)

12. I. Antoniadis, Phys. Lett. B 246, 377 (1990)

13. N. Arkani-Hamed, S. Dimopoulos, G. Dvali, Phys. Lett. B 429, 263 (1998)

14. I. Antoniadis, N. Arkani-Hamed, S. Dimopoulos, G. Dvali, Phys. Lett. B 436, 257 (1998)

15. G. Shiu, S.-H. Type, Phys. Rev. D 58, 106007 (1998)

16. L. Randall, R. Sundrum, Phys. Rev. Lett 83, 3370 (1999)

17. T. Appelquist, H.-C. Cheng, B.A. Dobrescu, Phys. Rev. D 64, $035002(2001)$

18. T.G. Rizzo, Phys. Rev. D 64, 095010 (2001)

19. C. Macesanu, C.D. McMullen, S. Nandi, Phys. Rev. D 66, 015009 (2002)

20. G. Aad et al., ATLAS Collaboration. Phys. Lett. B 716, 1 (2012)
21. S. Chatrchyan et al., CMS Collaboration. Phys. Lett. B 716, 30 (2012)

22. P. Langacker, Phys. Rep. 72, 185 (1981)

23. R.W. Robinett, J.L. Rosner, Phys. Rev. D 25, 3036 (1982)

24. R.W. Robinett, J.L. Rosner, Phys. Rev. D 27, 679(E) (1983)

25. R.W. Robinett, J.L. Rosner, Phys. Rev. D 26, 2396 (1982)

26. P. Langacker, R.W. Robinett, J.L. Rosner, Phys. Rev. D 30, 1470 (1984)

27. D. London, J.L. Rosner, Phys. Rev. D 34, 1530 (1986)

28. J.L. Hewett, T.G. Rizzo, Phys. Rep. 183, 193 (1989)

29. L.E. Ibáñez, J.E. Kim, H.P. Nilles, F. Quevedo, Phys. Lett. B 191, $282(1987)$

30. J.A. Casas, E.K. Katehou, C. Muñoz, Nucl. Phys. B 317, 171 (1989)

31. M. Cvetič, P. Langacker, Phys. Rev. D 54, 3570 (1996)

32. M. Cvetič , P. Langacker, Mod. Phys. Lett. A 11, 1247 (1996)

33. N. Arkani-Hamed, A.G. Cohen, H. Georgi, Phys. Lett. B 513, 232 (2001)

34. N. Arkani-Hamed, A.G. Cohen, E. Katz, A.E. Nelson, T. Gregoire, J.G. Wacker, JHEP 08, 021 (2002)

35. T. Han, H.E. Logan, B. McElrath, L.-T. Wang, Phys. Rev. D 67, 095004 (2003)

36. M. Masip, A. Pomarol, Phys. Rev. D 60, 096005 (1999)

37. R. Casalbuoni, S. De Curtis, D. Dominici, R. Gatto, Phys. Lett. B 462, 48 (1999)

38. A. Delgado, A. Pomarol, M. Quirós, JHEP 01, 030 (2000)

39. K. Agashe, A. Delgado, M.J. May, R. Sundrum, JHEP 08, 050 (2003)

40. K. Agashe, H. Davoudiasl, S. Gopalakrishna, T. Han, G.-Y. Huang, G. Perez, Z.-G. Si, A. Soni, Phys. Rev. D 76, 115015 (2007)

41. R.N. Mohapatra, R.E. Marshak, Phys. Rev. Lett. 44, 1316 (1980)

42. R.N. Mohapatra, R.E. Marshak, Phys. Rev. Lett. 44, 1643(E) (1980)

43. C. Wetterich, Nucl. Phys. B 187, 343 (1981)

44. W. Buchmuller, C. Greub, P. Minkowski, Phys. Lett. B 267, 395 (1991)

45. M. Aoki, N. Oshimo, Phys. Rev. Lett. 84, 5269 (2000)

46. J. Erler, P. Langacker, T. Li, Phys. Rev. D 66, 015002 (2002)

47. M. Carena, A. Daleo, B.A. Dobrescu, T.M.P. Tait, Phys. Rev. D 70, 093009 (2004)

48. B. Körs, P. Nath, Phys. Lett. B 586, 366 (2004)

49. B. Körs, P. Nath, JHEP 12, 005 (2004)

50. B. Körs, P. Nath, JHEP 07, 069 (2005)

51. P.H. Chankowski, S. Pokorski, J. Wagner, Eur. Phys. J. C 47, 187 (2006)

52. D. Feldman, Z. Liu, P. Nath, Phys. Rev. D 75, 115001 (2007)

53. S. Khalil, J. Phys. G 35, 055001 (2008)

54. S. Khalil, A. Masiero, Phys. Lett. B 665, 374 (2008)

55. S. Khalil, Phys. Rev. D 82, 077702 (2010)

56. S. Iso, N. Okada, Y. Orikasa, Phys. Lett. B 676, 81 (2009)

57. P.V. Dong, H.T. Hung, T.D. Tham, Phys. Rev. D 87, 115003 (2013)

58. S. Kanemura, T. Matsui, H. Sugiyama, Phys. Rev. D 90, 013001 (2014)

59. P.V. Dong, Phys. Rev. D 92, 055026 (2015)

60. P.V. Dong, D.T. Si, Phys. Rev. D 93, 115003 (2016)

61. N. Okada, S. Okada, Phys. Rev. D 95, 035025 (2017)

62. C. Kownacki, E. Ma, N. Pollard, O. Popov, M. Zakeri, Eur. Phys. J. C 78, 148 (2018)

63. W. Chao, Eur. Phys. J. C 78, 103 (2018)

64. T. Nomura, H. Okada, JHEP 1801, 099 (2018)

65. T. Nomura, H. Okada, Phys. Rev. D 97, 015015 (2018)

66. L. Dhargyal, Eur. Phys. J. C 78, 150 (2018)

67. A. Leike, Phys. Rep. 317, 143 (1999)

68. P. Langacker, Rev. Mod. Phys. 81, 1199 (2009)

69. C. Patrignani et al., Particle Data Group. Chin. Phys. C 40, 100001 (2016)

70. G. Aad et al., ATLAS Collaboration. JHEP 1211, 138 (2012) 
71. S. Chatrchyan et al., CMS Collaboration. Phys. Lett. B 720, 63 (2013)

72. G. Aad et al., ATLAS Collaboration. Phys. Rev. D 90, 052005 (2014)

73. V. Khachatryan et al., CMS Collaboration. JHEP 1504, 025 (2015)

74. M. Aaboud et al., ATLAS Collaboration. Phys. Lett. B 761, 372 (2016)

75. M. Aaboud et al., ATLAS Collaboration. JHEP 1710, 182 (2017)

76. V. Khachatryan et al., CMS Collaboration. Phys. Lett. B 768, 57 (2017)

77. H.A. Gomes, J. Math. Phys. 52, 082501 (2011)

78. Mikio Nakahara, Geometry (Institute of Physics Publishing, London, Topology and Physics, London, 2003)

79. S. Schael et al., ALEPH and DELPHI and L3 and OPAL and LEP Electroweak Collaborations. Phys. Rep. 532, 119 (2013)

80. S.G. Porsev, K. Beloy, A. Derevianko, Phys. Rev. Lett. 102, 181601 (2009)

81. S.G. Porsev, K. Beloy, A. Derevianko, Phys. Rev. D 82, 036008 (2010)

82. C.S. Wood, S.C. Bennett, D. Cho, B.P. Masterson, J.L. Roberts, C.E. Tanner, C.E. Wieman, Science 275, 1759 (1997)
83. S.C. Bennett, C.E. Wieman, Phys. Rev. Lett. 82, 2484 (1999)

84. S.C. Bennett, C.E. Wieman, Phys. Rev. Lett. 82, 4153(E) (1999)

85. S.C. Bennett, C.E. Wieman, Phys. Rev. Lett. 83, 889(E) (1999)

86. W.J. Marciano, A. Sirlin, Phys. Rev. D 27, 552 (1983)

87. W.J. Marciano, A. Sirlin, Phys. Rev. D 29, 75 (1984)

88. W.J. Marciano, A. Sirlin, Phys. Rev. D 31, 213(E) (1985)

89. W.J. Marciano, J.L. Rosner, Phys. Rev. Lett. 65, 2963 (1990)

90. W.J. Marciano, J.L. Rosner, Phys. Rev. Lett. 68, 898(E) (1992)

91. R. Diener, S. Godfrey, I. Turan, Phys. Rev. D 86, 115017 (2012)

92. F. Jegerlehner, Springer Tracts Mod. Phys. 274, 611 (2017)

93. G.W. Bennett et al., Muon $g-2$ Collab. Phys. Rev. D 73, 072003 (2006)

94. B.E. Lautrup, A. Peterman, E. de Rafael, Phys. Rep. 3C, 193 (1972)

95. J.P. Leveille, Nucl. Phys. B 137, 63 (1978)

96. F. Petriello, S. Quackenbush, Phys. Rev. D 77, 115004 (2008)

97. T. G. Rizzo, arXiv: hep-ph/0610104 